\title{
GRIPT: a novel case-control analysis method for Mendelian disease gene discovery
}

\author{
Jun Wang ${ }^{1,2}$, Li Zhao ${ }^{2,3}$, Xia Wang ${ }^{2,4}$, Yong Chen ${ }^{5}$, Mingchu Xu ${ }^{1,2}$, Zachry T. Soens ${ }^{1,2}$, Zhongqi Ge ${ }^{1,2}$, \\ Peter Ronghan Wang ${ }^{2}$, Fei Wang ${ }^{5}$ and Rui Chen ${ }^{1,2,3^{*}}$
}

\begin{abstract}
Despite rapid progress of next-generation sequencing (NGS) technologies, the disease-causing genes underpinning about half of all Mendelian diseases remain elusive. One main challenge is the high genetic heterogeneity of Mendelian diseases in which similar phenotypes are caused by different genes and each gene only accounts for a small proportion of the patients. To overcome this gap, we developed a novel method, the Gene Ranking, Identification and Prediction Tool (GRIPT), for performing case-control analysis of NGS data. Analyses of simulated and real datasets show that GRIPT is well-powered for disease gene discovery, especially for diseases with high locus heterogeneity.
\end{abstract}

Keywords: Mendelian disease, Disease gene prioritization, Cohort analysis, Locus heterogeneity, Next-generation sequencing

\section{Background}

Mendelian diseases refer to the diseases caused by mutations in a single gene and are inherited following Mendel's laws. It was estimated that approximately $0.4 \%$ of live-born individuals have clinically recognizable Mendelian phenotypes by early adulthood, and about eight million children worldwide are born each year with a serious genetic condition leading to disability or threatening lives $[1,2]$. Identification of Mendelian disease-causing genes can directly improve molecular diagnosis and genetic counseling and also provide new insights into the genetic and pathogenic mechanisms underlying the diseases, laying the foundations for developing preventive and therapeutic methods for patients $[3,4]$.

Traditional strategies for Mendelian disease gene discovery are primarily family-based approaches. Linkage analysis was widely used for mapping genes underlying dominant inherited diseases, while homozygosity mapping was successfully applied on recessive inherited

\footnotetext{
* Correspondence: ruichen@bcm.edu

${ }^{1}$ Human Genome Sequencing Center, Baylor College of Medicine, Houston, TX 77030, USA

${ }^{2}$ Department of Molecular and Human Genetics, Baylor College of Medicine, Houston, TX 77030, USA

Full list of author information is available at the end of the article
}

diseases in consanguineous families [5-9]. However, family-based strategies are limited by the availability of multi-member families and cannot be effectively applied to the sporadic cases of rare diseases. On the other hand, as the recent advances in next-generation sequencing (NGS) technology and the establishment of large patient cohorts, case-control analysis of patient NGS data has provided powerful alternatives in novel disease gene discovery $[7,10]$. Case-control analysis methods typically map candidate genes mutated in multiple affected patients (i.e., cases) but in wildtype form in unaffected individuals (i.e., controls). However, it remains challenging for these methods to distinguish the candidate disease genes from the genes with large numbers of rare benign variants (e.g., the highly mutable genes). Furthermore, the enormous amount of data generated by NGS brings huge analytical and computational burdens, which requires algorithms that can efficiently search through large numbers of whole genome/exome data and reliably detect the true signal of the disease gene from the massive background noise.

Previously, for case-control analysis, association tests were developed to identify the relation between genotypes and the phenotype, such as rare variant vs. common complex diseases. Particularly, the group-wise (i.e., 
gene/locus-based) association tests have been applied to enrich association signals and reduce the penalty for multiple testing. For example, "burden tests" or "collapsing methods," such as Combined Multivariate and Collapsing (CMC) [11], Cohort Allelic Sums Test (CAST) [12], and Weighted-Sum method [13], aggregate prioritization information across multiple variants within a genetic region. Furthermore, the kernel-based methods, such as Sequence Kernel Association Test (SKAT) [14] and Kernel-Based Adaptive Clustering (KBAC) [15], take into account the different effect direction and magnitude of variants within a locus when grouping the variants together. However, these methods were not originally designed for Mendelian diseases. Moreover, most of these methods are mainly based on the allele frequency differences and take little account of the functional predictions of individual alleles. In 2011, a case-control analysis method named Variant Annotation, Analysis and Search Tool (VAAST) and, later, an upgraded version, VAAST2, were developed for disease gene discovery of Mendelian disorders [16, 17]. VAAST/ VAAST2 measures the aggregative impact of variants within a gene based on the variant frequency differences between cases and controls and also considers the functional effects of variants by weighting amino acid substitution frequency and phylogenetic conservation $[16,17]$. However, VAAST/VAAST2 is prone to producing false positives, prioritizing the genes with large numbers of rare benign variants as the candidate disease genes. In addition, its specificity is greatly reduced when analyzing cohorts with high population stratification.

So far, 3532 genes underlying 5159 Mendelian phenotypes have been discovered, according to the Online Mendelian Inheritance in Man (OMIM) database (OMIM statistics, May 11, 2018) [18]. But the genes mutated in about $50 \%$ of the known Mendelian disorders remain elusive, and many more Mendelian phenotypes have not yet been recognized [10]. One main challenge is that the disease is often rare and genetically heterogeneous where each disease-causing gene only accounts for a very small proportion of patients with the disease [10]. To address this challenge, we developed a novel method, named the Gene Ranking, Identification and Prediction Tool (GRIPT), to identify Mendelian disease genes through analyzing genomic sequence data of patient-control datasets. By testing both simulated and real datasets, we demonstrated that GRIPT has excellent sensitivity and specificity in identifying known and novel disease genes. It significantly outperforms other state-of-the-art tools in discovering disease genes underlying patient cohorts with high locus heterogeneity. Moreover, GRIPT is quite robust and less affected by potentially confounding factors, such as patient cohort size, population stratification in cohorts, and cutoff of variant frequency filtering.

\section{Results}

The framework of GRIPT

GRIPT is specifically designed for Mendelian disease gene discovery through prioritizing genes with significantly higher deleterious mutation load in patients than controls as the candidate genes. In implementation, GRIPT first ranks the variants within each gene for every individual in both patient and control cohorts according to the variant effect score provided by users, e.g., CADD score [19] (Fig. 1, see the "Methods" section). Based on the variant scores, a gene score is calculated for each gene measuring the deleterious mutation load of the gene in every individual under a given inheritance model, i.e., autosomal dominant (AD), autosomal recessive (AR), X-linked dominant (XD), or X-linked recessive (XR) model (see the "Methods" section). Then, a Fisher's test built upon the combination of a binomial test and a Wilcoxon rank sum test (WRST) is applied to compare the gene score distributions in patients and controls for each gene, and a significant $p$ value associated with the test statistic is assigned. This composite test is especially suitable to compare two highly skewed distributions with excesses of zero, such as the gene score distributions in the case and control cohorts (Fig. 2, see the "Methods" section) [20]. Finally, GRIPT compares and ranks all genes based on the test statistic of each gene (Fig. 1).

\section{Simulation analysis tests the sensitivity and specificity of GRIPT}

To evaluate the sensitivity and specificity of GRIPT, we simulated WES data for patient and control cohorts under both the AR and AD inheritance models based on the variant profile of the human genome in the ExAC database [21] (see the "Methods" section). To mimic the patient cohort with high disease-locus heterogeneity where a given disease gene only accounts for a small proportion of the patients, pathogenic mutations of the same gene were randomly selected from the Human Gene Mutation Database (HGMD) and spiked into a small proportion (e.g., $0.5 \%, 1 \%, 2 \%$, or $3 \%$, respectively) of individuals in the patient cohort (see the "Methods" section). The size of patient cohort was set at 600 and the control cohort at 5000. The simulation for each scenario was repeated 30 times. A genome-wide statistical significance level (GWSL) of $2.7 \times 10^{-6}$ was used as the significant $p$ value cutoff for multi-testing correction (given about 18,500 autosomal protein-coding genes annotated by RefSeq genes). The performance of GRIPT was measured with three parameters: (1) the ranking of the disease gene with spike-in pathogenic mutations, indicating the sensitivity of the tool; (2) the percentage of simulation runs in which the disease gene passes GWSL, indicating the statistical power of the tool; and (3) the number of significant autosomal candidate genes, indicating the 


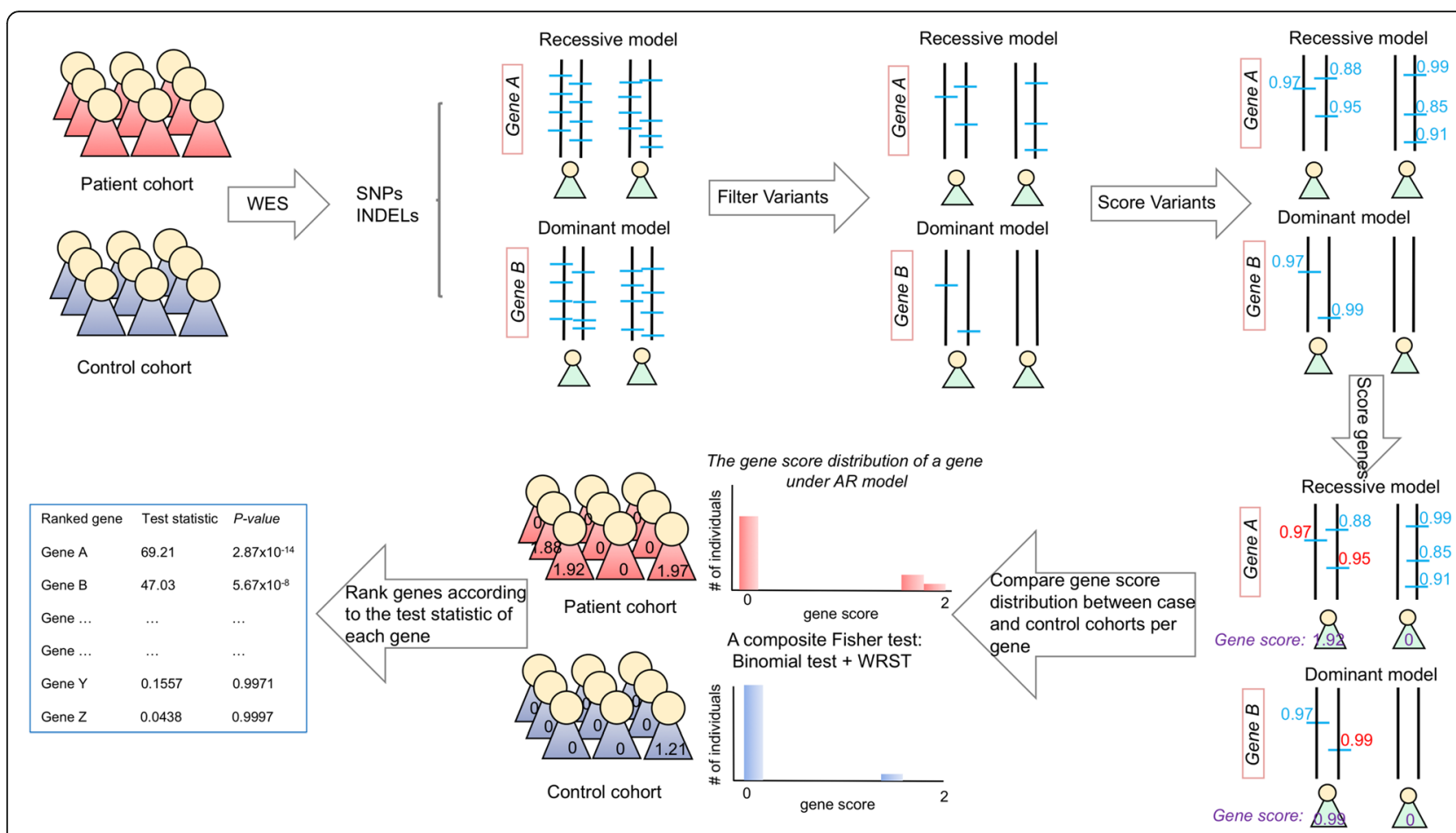

Fig. 1 The logic flowchart of GRIPT. First, the samples of the case and control cohorts will be collected and be subjected to NGS, e.g., WES. After variant calling, the known common and/or benign variants will be filtered out based on the variant annotation and their allele frequency in large databases of normal populations and internal databases. Thus, for each gene, only a few rare variants will be left. Then, GRIPT will annotate and rank the deleteriousness of each variant, e.g., using CADD score. Based on the variant scores, a gene score will be calculated to measure the deleterious mutation load of each gene in every individual according to a given inheritance model (see the "Methods" section). Next, a Fisher's test built upon the combination of a binomial test and a Wilcoxon rank sum test (WRST) will be calculated to measure the difference of gene score distributions between patient cohort and control cohort for each gene, and a significant $p$ value associated with the test statistic will be assigned. This composite test is especially well suited to measure the difference of two highly skewed distributions with excesses of 0 , such as the gene score distribution in the patient/control cohort computed by GRIPT (Fig. 2). Finally, according to the test statistic of each gene, GRIPT compares and ranks all genes

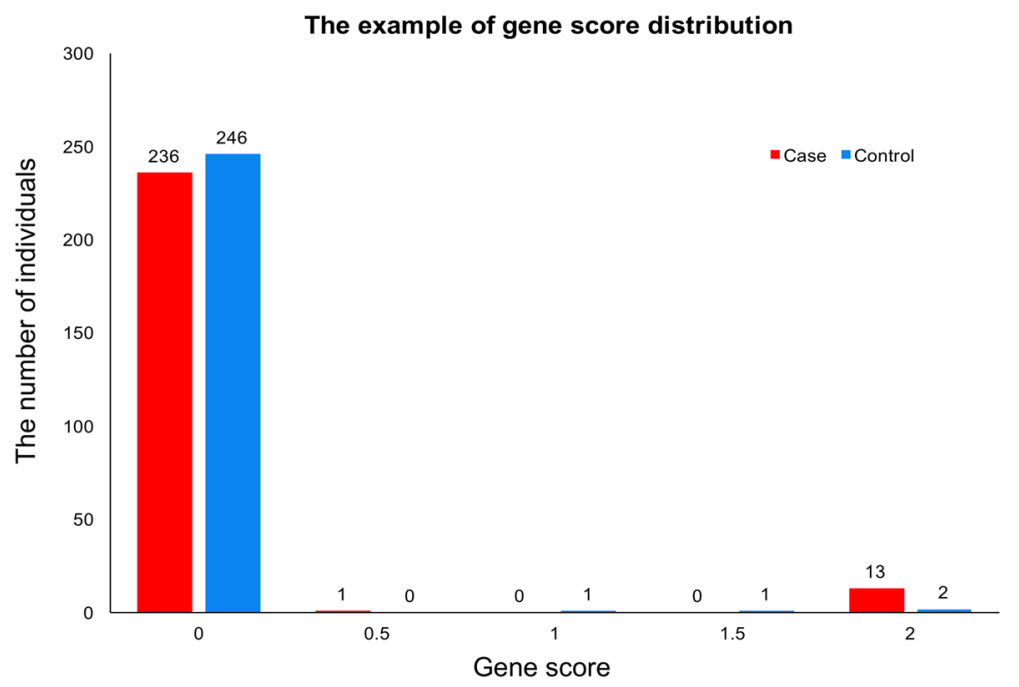

Fig. 2 The example of gene score distribution. This figure shows the gene score distributions of USH2A in a retinal disease cohort of 250 patients (in red) and in a control cohort of 250 individuals (in blue). $X$ axis: the gene score of USH2A per individual. $Y$ axis: the numbers of patients or controls with the corresponding score. Like the gene USH2A, the gene score distributions of most genes are highly skewed with excesses of zeros 
specificity of the tool. Furthermore, the performance of GRIPT was compared with four popular cohort analysis tools, including the Mendelian disease gene finder, VAAST2, and three group-wise association tests, the CMC (burden test), SKAT, and KBAC (kernel model), on the same datasets [11, 14, 15, 17, 22].

\section{The sensitivity and specificity of GRIPT under the AR and $A D$ models}

To test the performance of GRIPT in identifying AR disease gene, RPE65 was used as an example. RPE65 is a well-studied gene with mutations known to cause AR Leber congenital amaurosis (LCA) and retinitis pigmentosa (RP) [23-25]. The performance of the four tests is summarized in Fig. 3 and Additional file 1: Table S1. Figure $3 \mathrm{a}-\mathrm{c}$ and Additional file 1: Table S1 demonstrate that GRIPT has great sensitivity and specificity in detecting RPE65, even when the proportion of RPE65 patients was very low, mimicking the scenario of patient cohort with high locus heterogeneity. When the RPE65 patient proportion was as low as $0.5 \%$, GRIPT ranked RPE65 on average sixth, achieving $66.67 \%$ power. When the RPE65 patient proportion reached $\geq 1 \%$, GRIPT ranked RPE65 first in all trials with $100 \%$ power. Across the range of RPE65 patient proportions, GRIPT identified on average three significant candidates per simulation. In contrast, with a low proportion of RPE65 patients, the other four algorithms had significantly lower sensitivity and power than GRIPT (WRST, $p$ value, see Additional file 1: Table $\mathrm{S} 1)$. For example, when the RPE65 patient proportion was $\leq 1 \%$, the powers of the other four tests were $\leq 10 \%$ and the mean rank of RPE65 was between 38 and 3068 . Each of the other four methods identified on average zero or one significant candidate gene.

In parallel, the performance of GRIPT in identifying $\mathrm{AD}$ disease gene was tested using TINF2 as an example. TINF2 is a known, disease-causing gene of AD Revesz syndrome and dyskeratosis congenita [26-28]. As shown in Fig. 3d-f and Additional file 1: Table S1, GRIPT lacked power when the TINF2 patient proportion was very low, but its performance was greatly improved as the TINF2 patient proportion increased. Specifically, as TINF2 patient proportion increased from 0.5 to $1 \%$, the power of GRIPT increased from 3.33 to $53.33 \%$. When

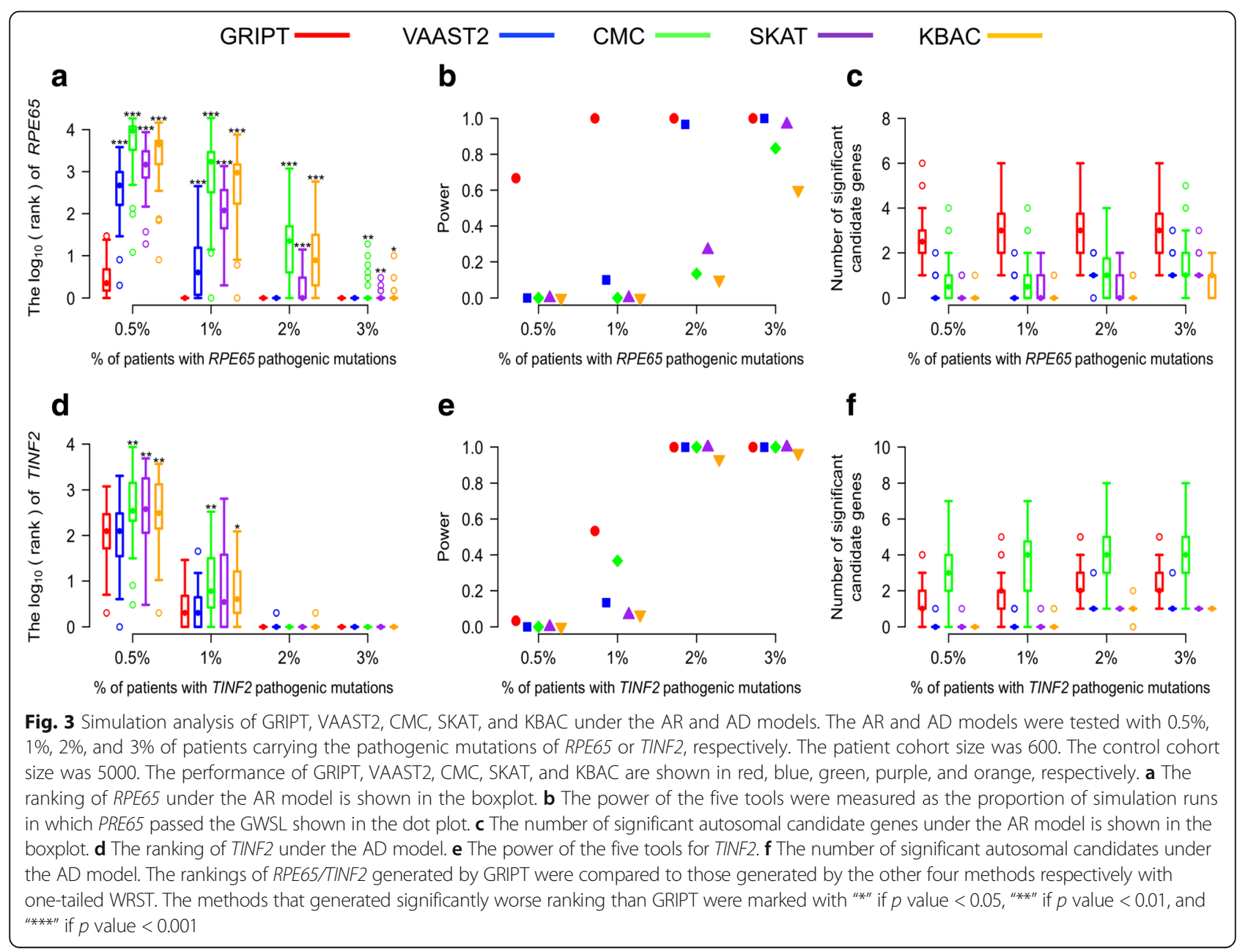


the TINF2 patient proportion reached $\geq 2 \%$, TINF2 was always ranked first by GRIPT with $100 \%$ power. On average, GRIPT identified about two significant candidate genes. In comparison, the other four methods had significantly worse performance than GRIPT (WRST, $p$ value, see Additional file 1: Table S1). For example, when TINF2 patient proportion increased from 0.5 to $1 \%$, the power of VAAST2 increased from 0 to $13.33 \%$, CMC from 0 to $36.67 \%$, SKAT from 0 to $6.67 \%$, and KBAC from 0 to $6.67 \%$.

\section{Benchmark on 400 randomly selected known disease genes} To further expand the evaluation of GRIPT, we performed simulation using 400 Mendelian disease-causing genes randomly selected from the OMIM database, including $200 \mathrm{AR}$ and $200 \mathrm{AD}$ disease genes. For each gene, we simulated the patient cohorts with a size of 600 and used the same simulated control cohort with a size of 5000. The results are summarized in Fig. 4 and Additional file 1: Table S2.

Consistent with the results for RPE65, GRIPT showed outstanding sensitivity and specificity in detecting the 200 AR genes even when the proportion of patients attributed to the same disease gene was very low (Fig. 4a-c). Consistently, VAAST2, CMC, SKAT, and KBAC showed significantly worse performance than GRIPT when patient cohort had high locus heterogeneity (Fig. 4a-c, WRST, $p$ value, see Additional file 1: Table S2). When the proportion of patients attributed to the same disease gene was as low as $0.5 \%$, the disease genes were ranked on average 24th by GRIPT achieving $52.5 \%$ power, whereas the other four methods had $0 \%$ power. When the patient proportion equaled to $1 \%$, the disease genes were ranked on average first by GRIPT with $97 \%$ power. In contrast, the power of the other four methods was between 0.5 and $11.5 \%$. When the patient proportion reached $\geq 2 \%$, the disease genes were always ranked first by GRIPT with $100 \%$ power. In comparison, the power of the four methods was between 11.5 and $97.5 \%$. Across the range of patient proportions, GRIPT identified on average one significant candidate gene compared to zero or one candidate by each of the other four methods.

Consistent with the results of TINF2, the overall performance of GRIPT was better than or comparable to the other four methods in detecting the $200 \mathrm{AD}$ genes

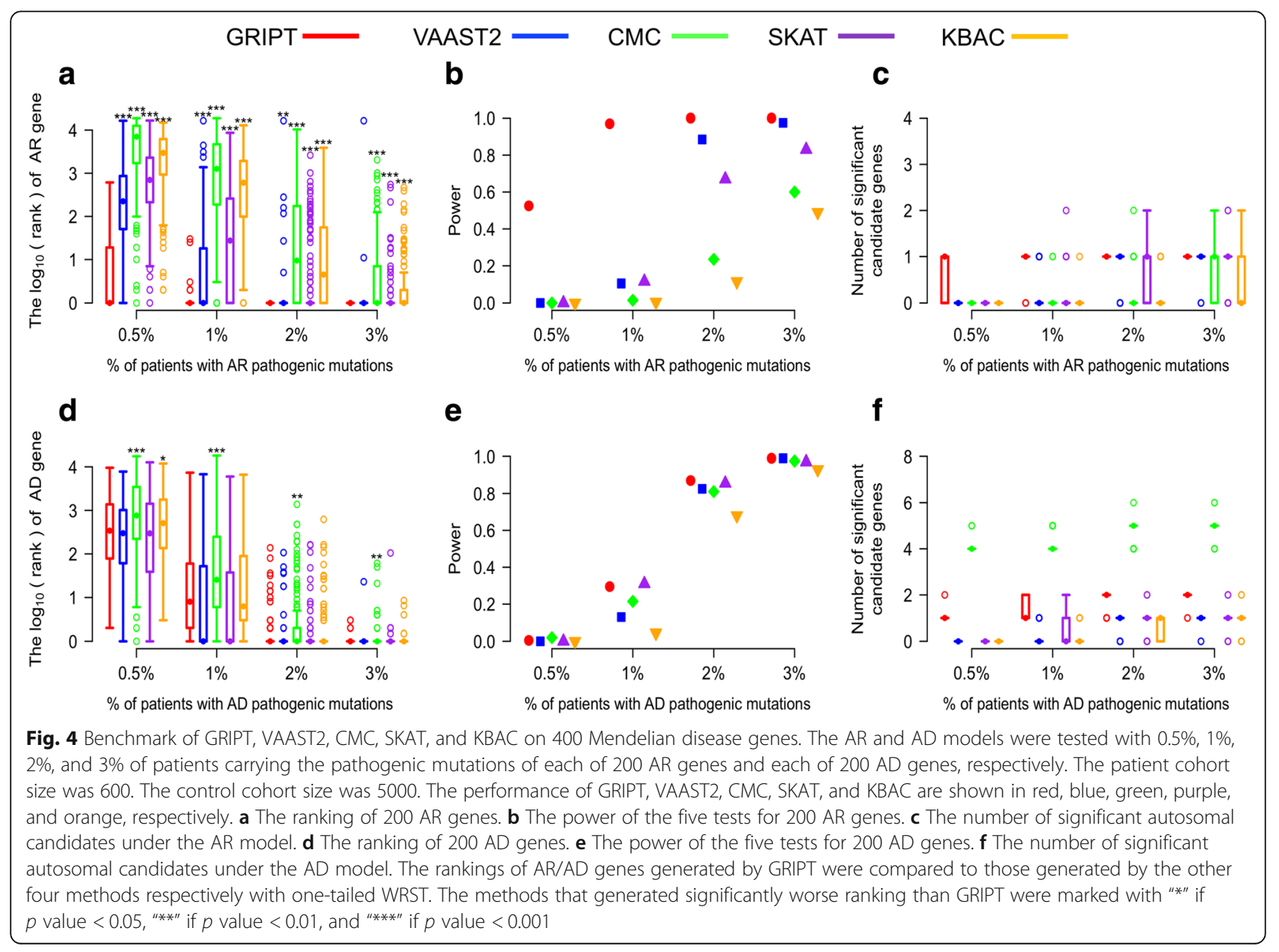


(WRST, $p$ value, see Additional file 1: Table S2). When the proportion of patients attributed to the same disease gene was $\leq 1 \%$, GRIPT and the other four tests have very low power, i.e., $\leq 29.5 \%$ for GRIPT, $\leq 13 \%$ for VAAST2, $\leq 21.5 \%$ for $\mathrm{CMC}, \leq 31 \%$ for SKAT, and $\leq 4.5 \%$ for KBAC (Fig. $4 \mathrm{~d}-\mathrm{f}$ ). When the patient proportion attributed to the same gene increased to $2 \%$, the disease genes were ranked on average third by GRIPT with $87 \%$ power. In comparison, the power of the other four tests was between 68 and $85.5 \%$. When the patient proportion reached 3\%, the disease genes were ranked first in $97.5 \%$ of simulations by GRIPT with $99 \%$ power. Comparably, the power of the other four tests increased to $93-99 \%$. Across the range of patient proportions, on average, one to two significant candidate genes were identified by GRIPT compared to between zero and five candidates by the other four methods.

\section{Simulations suggest GRIPT is highly robust}

The performance of case-control cohort analysis can be potentially impacted by several confounding factors, such as patient cohort size, population stratification, and the cutoff of variant filtering frequency, and the control cohort size. To assess their impact, we performed simulations using RPE65 and TINF2 as examples under the $\mathrm{AR}$ and $\mathrm{AD}$ models, respectively, and compared GRIPT with VAAST2, CMC, SKAT, and KBAC using the same datasets under each scenario. In addition, we tested the effect of different variant score systems on the performance of GRIPT.

\section{The sample size of the patient cohort}

We simulated the patient cohorts in a range of sizes, i.e., $50,100,300,600$, and 800 , with $2 \%$ of patients carrying the pathogenic mutations of the same disease genes, and control cohorts with a size of 5000 . The results are summarized in Fig. 5 and Additional file 1: Table S3.

As shown in Fig. 5a-c, under the AR model, GRIPT maintains high sensitivity for patient cohorts with a variety of sizes and high locus heterogeneity although its specificity decreased for small patient cohorts with high locus heterogeneity. In comparison, the other four methods performed significantly worse than GRIPT under the same situations (WRST, $p$ value, see Additional file 1: Table S3). Specifically, as the patient cohort size increased from 50 to 300 with $2 \%$ of patients carrying the RPE65 pathogenic mutations, the mean rank of RPE65 increases from 31 to 1 by GRIPT with $100 \%$

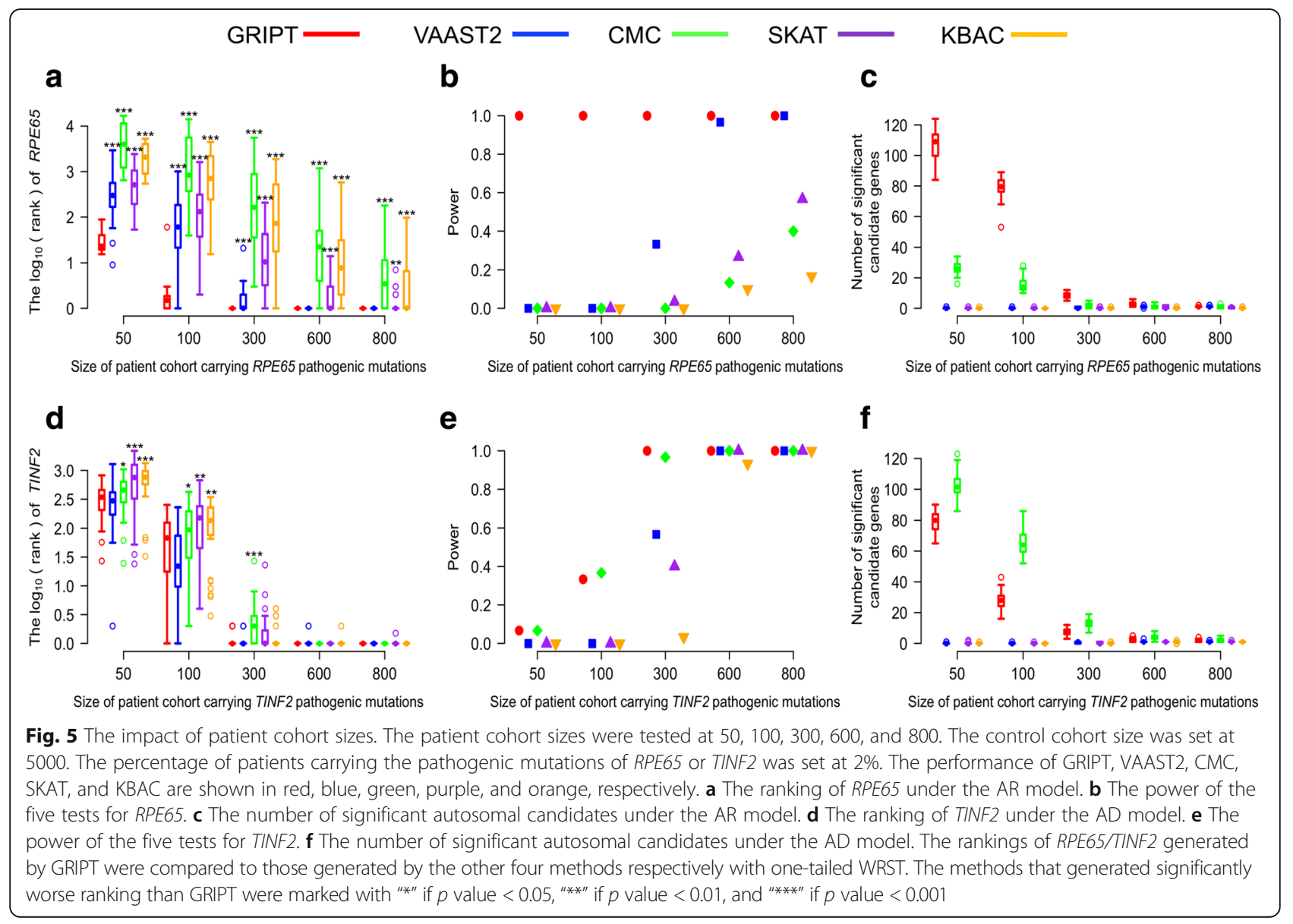


power. The number of significant candidates identified by GRIPT decreased from 107 to 8 . When the patient cohort size reached $\geq 300$, GRIPT always ranked RPE65 first with $100 \%$ power. The average number of significant candidates decreased to between one and eight. In contrast, the power of the other four methods was $0 \%$ when the patient cohort size is $<300$. When the patient cohort size reached $\geq 300$, the power was $33.33-100 \%$ for VAAST2, 0-40\% for CMC, 3-56.67\% for SKAT, and $0-16.67 \%$ for KBAC. And the average number of significant candidates identified by each of the four methods was between 0 and 26 .

Under the AD model, when patient cohort was small and had high locus heterogeneity, GRIPT had low sensitivity and specificity, but its performance was greatly improved as the patient cohort size increased (Fig. 5d-f). The other four methods performed comparably or significantly worse under the same scenarios (Fig. $5 \mathrm{~d}-\mathrm{f}$, WRST, $p$ value, see Additional file 1: Table S3). Specifically, when the patient cohort size increased from 50 to 100 with $2 \%$ of patients attributed to TINF2, the power of GRIPT increased from 6.67 to $33.33 \%$ and the average number of significant candidates decreased from 79 to 28 . When the patient cohort size increased to $\geq 300$, TINF2 was ranked on average first by GRIPT with 100\% power. The average number of significant candidates by GRIPT was between two and eight. In comparison, when the patient cohort size is $<300$, the power increased from 6.67 to $36.67 \%$ for $\mathrm{CMC}$ and remained at $0 \%$ for VAAST2, SKAT, and KBAC. When the patient cohort size reached $\geq 300$, the power was between 3.33 and $100 \%$ for the four tests. The average number of significant candidates by each of the four tests was between 0 and 103.

\section{Population stratification of cohorts}

It was observed that the variant spectrum of a disease gene is different among populations with different ethnicities and that high-population stratification could impair the performance of cohort analysis [16]. To test the impact of population stratification on GRIPT, we simulated patient cohorts as an admixture of African and Latino individuals and control cohorts with Latino individuals only, based on the allele frequency in ExAC database with corresponding ethnicity (see the "Methods" section). The unmatched proportion between case and control cohorts was simulated at $0 \%, 20 \%, 40 \%, 60 \%, 80 \%$, and $100 \%$. The size of patient cohort was set at 500 and the control cohort at 5000. The proportion of patients carrying the pathogenic mutations of the same gene was set at $1 \%$. The results are summarized in Fig. 6 and Additional file 1: Table S4.

As shown in Fig. 6a-f, the sensitivity and specificity of GRIPT slightly decreased as unmatched ethnicity proportion between cases and controls increased. However, GRIPT is significantly less affected by population stratification than the other four methods even when patient cohort had high locus heterogeneity (WRST, $p$ value, see Additional file 1: Table S4). Specifically, under the AR model, as the unmatched ethnicity proportion between patients and controls increased from 0 to $100 \%$ (namely, from the completely matched to the completely unmatched), the mean rank of RPE65 dropped from 1 to 32 by GRIPT but always with $100 \%$ power (Fig. $6 a-c$ ). Specificity was reduced as the average number of significant candidate genes increased from 2 to 111 (Fig. 6ac). In comparison, the powers of CMC, SKAT, and KBAC were between 0 and $20 \%$. The average number of significant candidate genes increased from 1 to 1929 for CMC, from 0 to 2603 for SKAT, and from 0 to 1921 for KBAC. In addition, as the unmatched ethnicity proportion increased, the running time for VAAST2 dramatically increased (e.g., needs 120-240 h with five parallel CPUs to finish one simulation run). Therefore, VAAST2 was only tested for the unmatched ethnicity proportion ranging from 0 to $60 \%$. Under those scenarios, the power of VAAST2 was between 10 and 26.7\%. The average number of significant candidate genes identified by VAAST2 increased from 0 to 1502.

Under the AD model, GRIPT is also significantly less affected by population stratification (WRST, $p$ value, see Additional file 1: Table S4). As the unmatched ethnicity proportion increased from 0 to $100 \%$, the mean rank of TINF2 dropped from two to nine by GRIPT with $96.67-$ $100 \%$ power (Fig. $6 \mathrm{~d}-\mathrm{f}$ ). The mean number of significant candidate genes increased from 3 to 19. In comparison, the mean rank of TINF2 dropped from 3 to 75 for VAAST2, from 7 to 57 for CMC, from 44 to 166 for SKAT, and from 3 to 33 for KBAC. The power was 0 13.33\% for VAAST2, 53.33-66.67\% for CMC, $0-3.33 \%$ for SKAT, and $0-6.67 \%$ for KBAC. The average number of significant candidate genes increased from zero to five for VAAST2, from 4 to 35 for $\mathrm{CMC}$, from zero to two for SKAT, and from zero to one for KBAC. (Fig. 6d-f).

\section{Variant frequency filtering}

Mendelian disease-causing mutations are expected to be very rare in the population, and common human variants are likely benign for rare Mendelian diseases. Therefore, to reduce the analysis/computation complexity, variants from WES are conventionally first filtered out common human genome variants based on allele frequency in a large database of human genome variants, e.g., gnomAD and ExAC [21]. To mimic this scenario, the above patient and control cohorts were simulated using the variants whose maximum population frequency is $\leq 0.5 \%$ in ExAC database for the AR model and whose maximum population frequency is $\leq 0.01 \%$ 


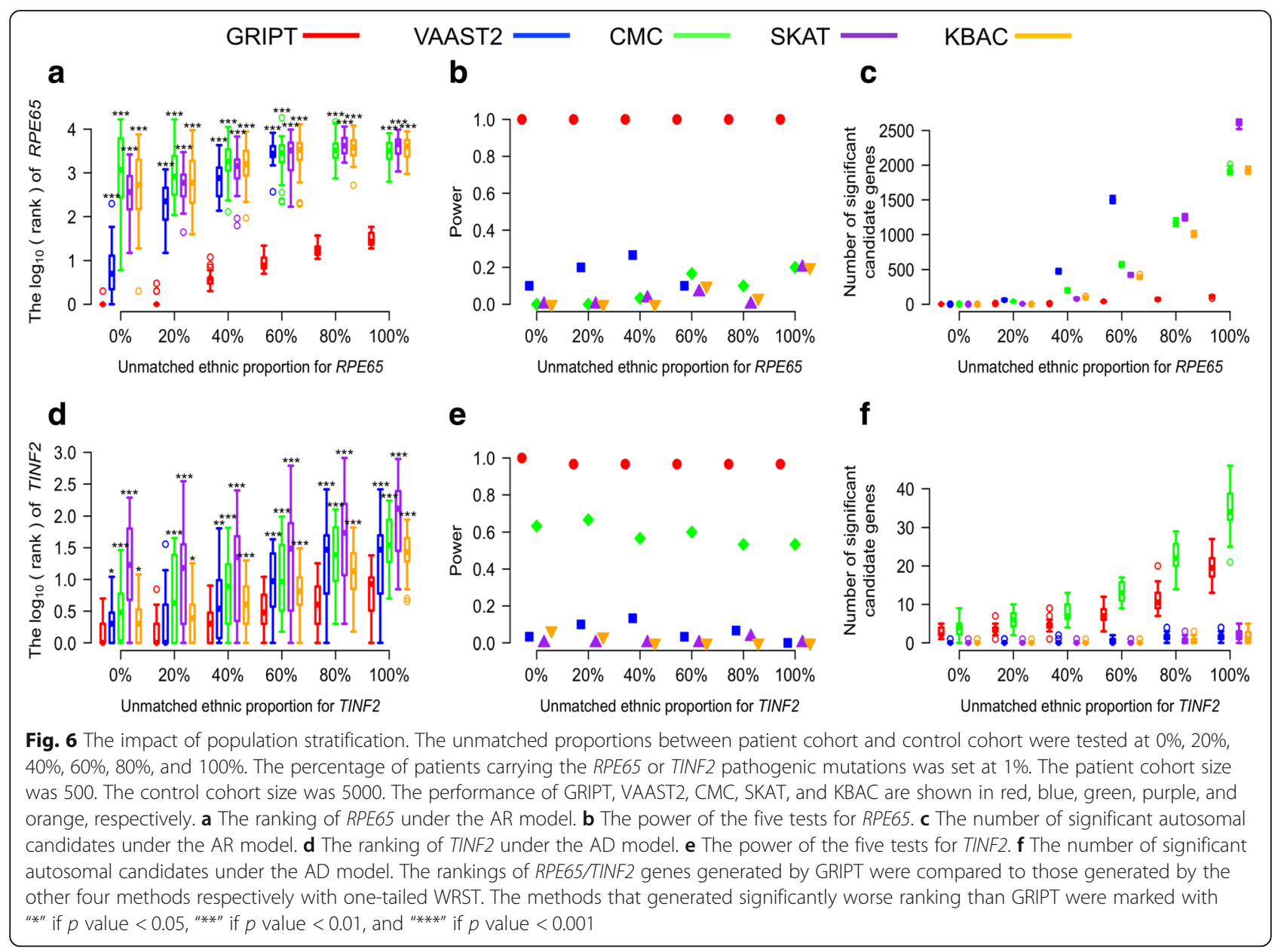

for the AD model. Here, we examined the impact of a relaxed (i.e., higher) frequency filtering cutoff on the disease gene identification methods. We simulated the WES data of patient and control cohorts using a range of variant frequency cutoffs respectively: $\leq 0.5 \%, \leq 1 \%$, and $\leq 2 \%$ for the AR model and $\leq 0.01 \%, \leq 0.5 \%$, and $\leq$ $1 \%$ for the AD model. The proportion of patients attributed to the same gene was set at $1 \%$. The size of patient cohort was set at 600 and control cohort at 5000. The results show that inclusion of more variants/noise per individual by using higher frequency filtering cutoff had little impact on GRIPT's performance under the AR model, but it reduced its power under the AD model. The performances of the other four methods were largely compromised and were significantly worse than or comparable to that of GRIPT (Fig. 7a-f, Additional file 1: Table S5).

Specifically, under the AR model, as the frequency filtering cutoff increased from 0.5 to $2 \%$, GRIPT ranked RPE65 first in $98.89 \%$ of the simulations, always achieving $100 \%$ power. The mean number of significant candidate genes was about three (Fig. $7 \mathrm{a}-\mathrm{c}$ ). In contrast, the ranking of RPE65 by the other four tests was largely decreased, with $\leq 10 \%$ power for VAAST 2 and $0 \%$ power for CMC, SKAT, and KBAC. Under the AD model, as the variant frequency cutoff increased from 0.01 to $1 \%$, the average rank of TINF2 dropped from 5 to 590 by GRIPT with power decreasing from 53.33 to around 3\%. The average number of significant candidate genes was between zero and two (Fig. $7 \mathrm{~d}-\mathrm{f}$ ). The power of VAAST2 decreased from 13.33 to $10 \%$, CMC from 36.67 to $0 \%$, SKAT from 6.67 to $0 \%$ for SKAT, and KBAC from 6.67 to $0 \%$.

\section{The effect of the control cohort size}

Theoretically, the variant spectrum of a gene in a large control cohort should be less biased and closer to the true distribution than that in a small control cohort. Thus, large control cohorts can better serve as the control/baseline, for example, to exclude the genes with large numbers of rare benign variants in population. To test the effect of control cohort size, we simulated smaller control cohorts with a size of 600 and used the previous case cohorts with a size of 600 to repeat the analysis. The results are summarized in Fig. 8 and Additional file 1: Table S6. 


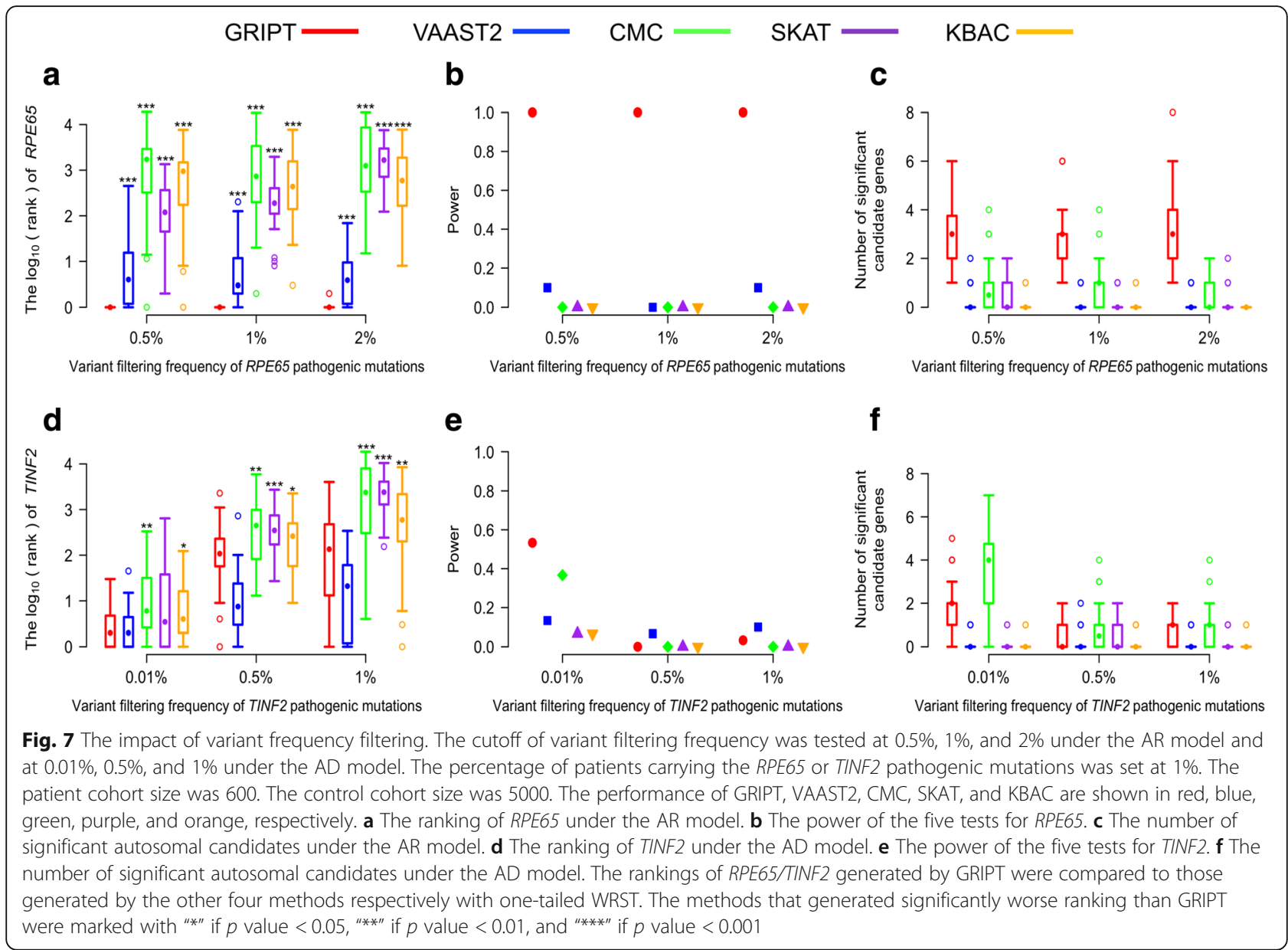

Under the AR model, GRIPT remained sensitive in ranking RPE65. When the RPE65 patient proportion increased from 0.5 to $\geq 2 \%$, the mean rank of RPE65 increased from 45 to 1 . However, the $p$ value of RPE65 did not pass the GWSL in any of the simulations, showing GRIPT with $0 \%$ power. Consistent with the results with larger control cohort, the other four tools performed significantly worse than GRIPT (Fig. 8a-c, WRST, $p$ value, see Additional file 1: Table S6). For example, when the RPE65 patient proportion equaled to $1 \%$, the mean rank of RPE65 was 981 for VAAST2, 6243 for CMC, 7611 for SKAT, and 2892 for KBAC. Similarly, the $p$ values of RPE65 from the other four tests did not pass the GWSL for the majority of the simulations either, shown as the test power below $13.33 \%$.

Under the AD model with the small control cohorts, the rankings of TINF2 by GRIPT and the other four methods were consistent to that with the large control cohorts (Fig. 8d-f, Additional file 1: Table S6). The five methods gave TINF2 a low ranking when the TINF2 patient proportion was low. But the ranking of TINF2 rose as the TINF2 patient proportion increased. When the TINF2 patient proportion increased to $3 \%$, all five methods ranked TINF2 to the top. However, similar to the results under the AR model, the $p$ value of TINF2 by the five methods did not pass the GWSL in the majority of the simulations under the AD model, shown as the power below $36.67 \%$ (Fig. 8d-f).

\section{The effect of different variant scoring systems}

To test whether the performance of GRIPT will be affected by different variant score systems, besides CADD score, we applied the Deleterious Annotation of genetic variants using Neural Networks (DANN) and Rare Exome Variant Ensemble Learner (REVEL) scores to annotate the variant scores in GRIPT respectively and repeated the aforementioned analyses. DANN scoring system shares the same feature set and training data as CADD (which was trained with a linear kernel support vector machine, SVM) but was trained with a non-linear deep neural network. DANN achieves about a 19\% relative reduction in the error rate and about a $14 \%$ relative increase in the area under the curve (AUC) metric over CADD's SVM methodology [29]. REVEL is an ensemble method for predicting the pathogenicity of missense variants by integrating the individual tools, including 


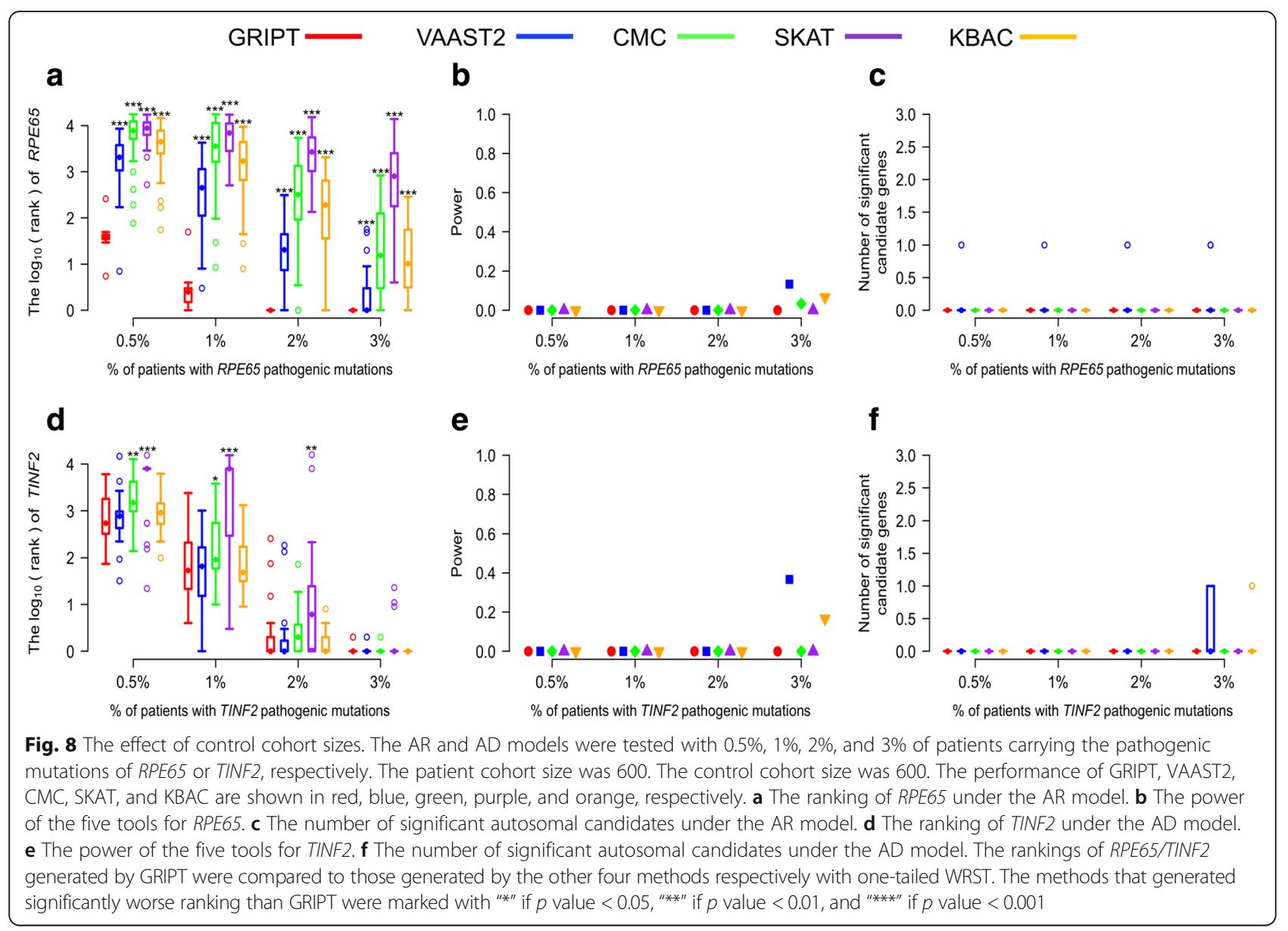

MutPred, FATHMM, VEST, PolyPhen, SIFT, PROVEAN, MutationAssessor, MutationTaster, LRT, GERP, SiPhy, phyloP, and phastCons. REVEL outperformed $\left(p<10^{-12}\right)$ individual tools and seven ensemble methods (i.e., MetaSVM, MetaLR, KGGSeq, Condel, CADD, DANN, and Eigen) in analyzing independent test sets and also showed the best performance for distinguishing pathogenic from rare neutral variants with allele frequencies $<0.5 \%$ [30]. As shown in Additional file 2: Figure S1-S4 and Additional file 1: Table S2-S5, the benchmark analysis with $400 \mathrm{AR}$ and AD genes, the analyses of the impacts of patient cohort size, population stratification, and variant frequency filtering all showed that the results based on DANN and REVEL scores are consistent with the previous results based on CADD score. The consistency based on different variant score systems demonstrated the reliability and robustness of the statistic test framework of GRIPT.

\section{Comparison to the traditional GWAS single variant test}

To compare the performance of GRIPT with the traditional GWAS single variant test, we simulated the basic scenario with $0.5-3 \%$ of patients carrying the pathogenic mutations of RPE65 and TINF2, respectively, and applied
GRIPT and Fisher's exact test to the data. As shown in Fig. 9 and Additional file 1: Table S1, Fisher's exact test performed much worse than GRIPT. Under the AR model, when the RPE65 patient proportion was $0.5 \%$, RPE65 was ranked on average sixth by GRIPT with $66.67 \%$ power. When the RPE65 patient proportion was $\geq 1 \%$, RPE65 was always ranked first by GRIPT with $100 \%$ power. In contrast, the average ranking of $R P E 65$ by Fisher's exact test was in the range of 890 to 32,000, always with $0 \%$ power (Fig. $9 \mathrm{a}-\mathrm{C}$ ). Under the AD model, as TINF2 patient proportion increased from 0.5 to $1 \%$, the power of GRIPT increased from 3.33 to $53.33 \%$. When the TINF2 patient proportion was $\geq 2 \%$, GRIPT always ranked TINF2 first with $100 \%$ power. In comparison, as the proportion of TINF2 patients increased, the average ranking of TINF2 by Fisher's exact test was improved from 12,675th to 23th, but the test power remained at $0 \%$ (Fig. $9 d-f$ ). The reasons may be as follows: (1) GRIPT is a gene-wise test that ranks the functional effects of variants and incorporates the Mendelian inheritance models to compute the gene score. In contrast, the traditional single variant test considers one variant in a gene each time and is mainly based on the allele frequency difference between cases and controls. 


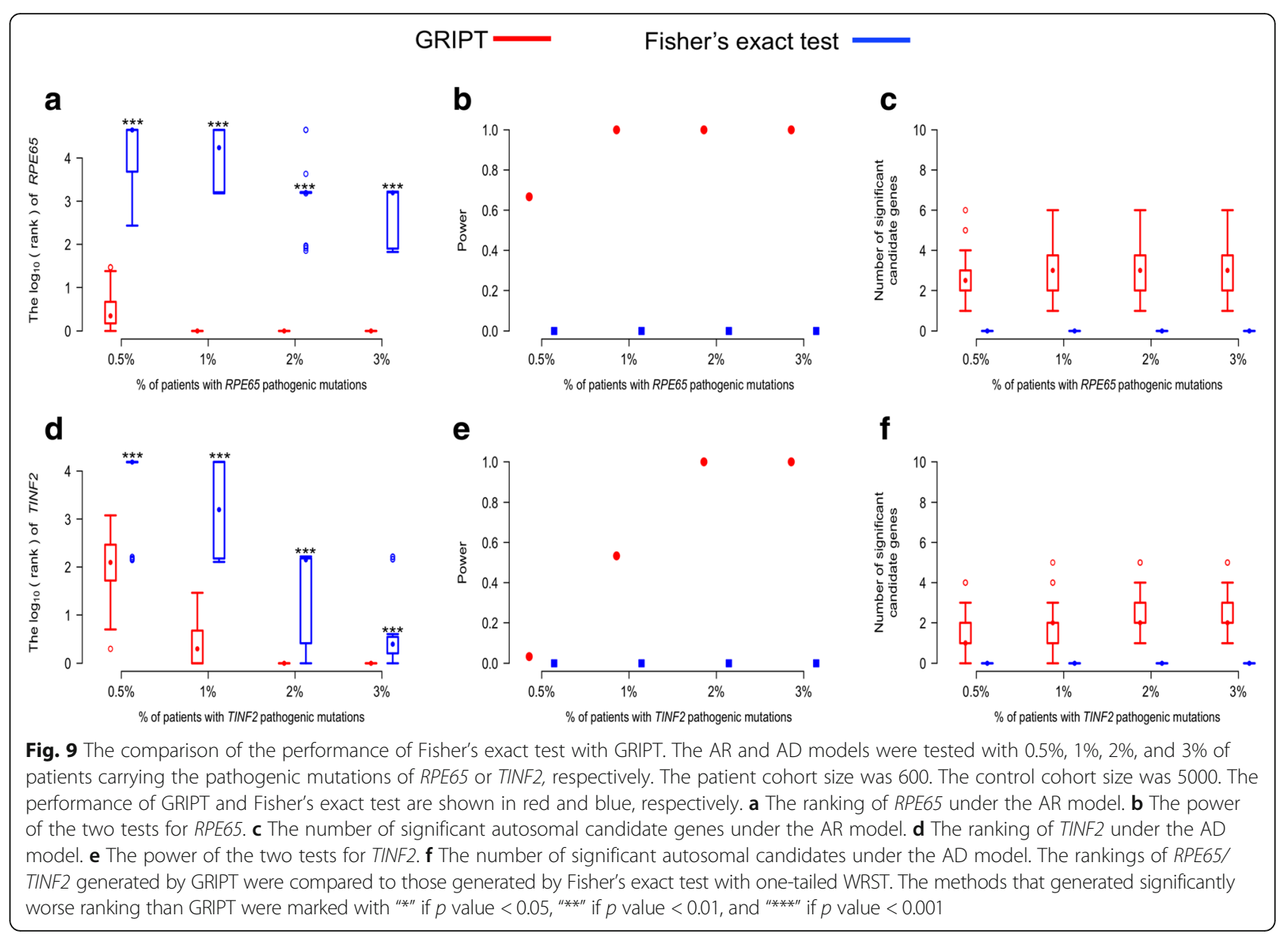

Thus, the single variant test does not have sufficient power to detect the heterogeneous rare deleterious variants in Mendelian disease cohorts, although it might be suitable for common complex diseases. (2) The multiple test correction requests a much more stringent $p$ value cutoff for the single variant test than the gene-wise GRIPT due to the larger number of tests applied in the single variant test than in GRIPT (i.e., variants vs. genes).

\section{The performance to detect genes with modest-effect variants}

To test the performance of GRIPT in detecting genes with modest-effect variants, we simulated the scenario of patients carrying allele complex composed of modest-effect mutations by concurrently spiking two known modest-effect mutations of $A B C A 4$, chr1:94476467:T>A (p.Asn1868Ile), and chr1: 94517254:C>G (p.Gly863Ala) [31] into the varying percentages (i.e., $0.5 \%, 1 \%, 2 \%$, and $3 \%$ ) of patients under the AR model. Both GRIPT and the collapsing test CMC were applied to the data. As shown in Fig. 10 and Additional file 1: Table S7, GRIPT significantly outperformed the collapsing test in detecting genes with modest-effect variants. The reason behind might be that GRIPT ranks the functional effects of variants and takes account of the Mendelian inheritance model to compute the gene score. In contrast, the collapsing test aggregates all the variants within a gene regardless of their functional effect and the Mendelian inheritance mode to compute the mutation burden, resulting in higher background noise; therefore, it is less sensitive and more prone to false positives for genes with large numbers of rare benign variants.

\section{Analysis of real patient cohort data displays GRIPT's excellent performance}

To further validate the performance of GRIPT, we applied it to real WES data of three different patient cohorts respectively, including a Leber's congenital amaurosis (LCA) cohort, a retinitis pigmentosa (RP) cohort, and a congenital disorder of glycosylation (CDG) cohort. Both the LCA cohort and RP cohort were composed of the patients carrying the pathogenic mutations of different genes, and the proportion of patients attributed to each disease gene was small. Furthermore, the patient ethnicity of the LCA cohort or RP cohort was an 


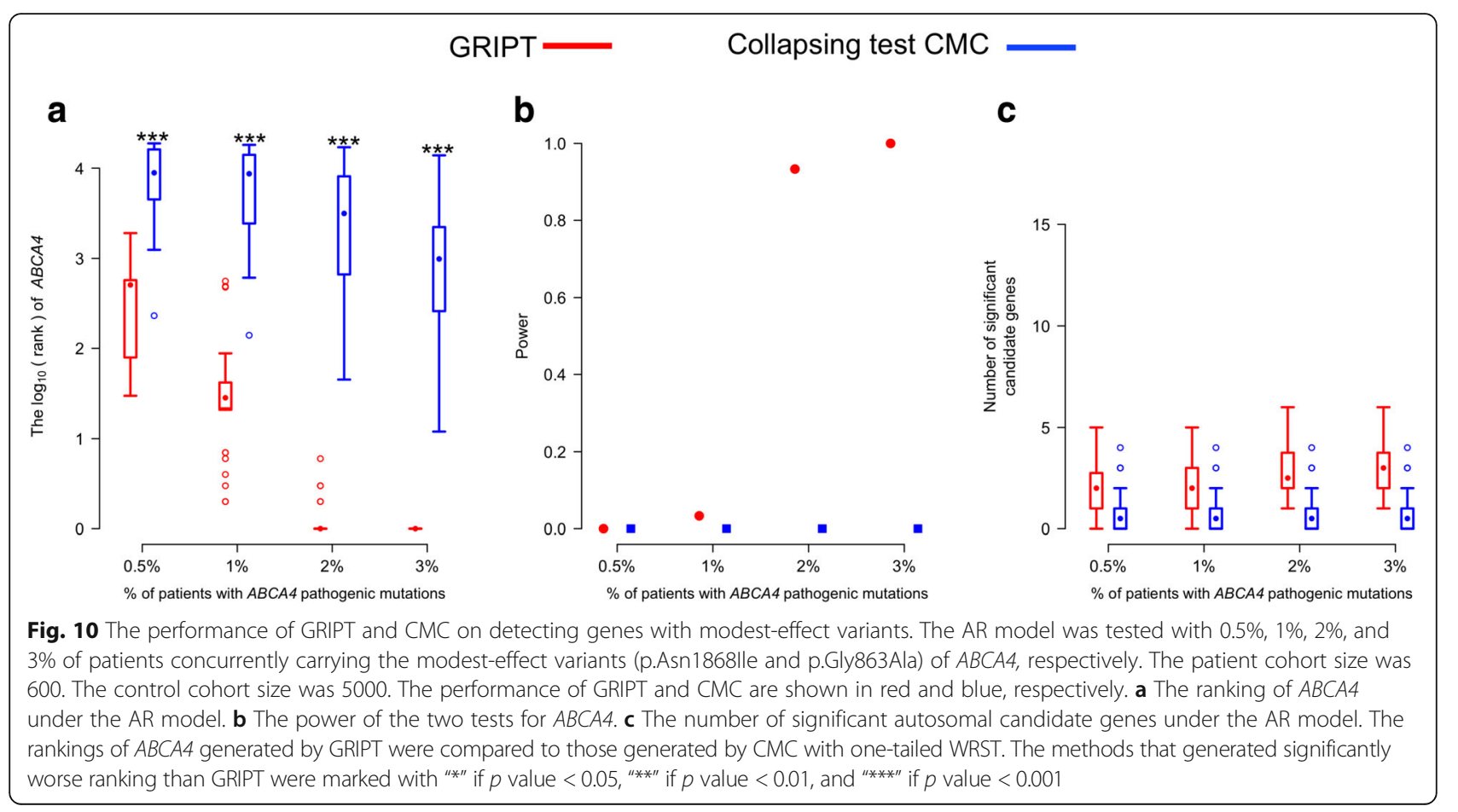

admixture of Caucasian, African American, Latino, and Asian, whereas the CDG cohort was composed of the patients all attributed to PGM3 from two families. The performance of GRIPT was also compared with VAAST2, CMC, SKAT, and KBAC on the same datasets.

\section{The LCA cohort}

LCA is a genetic heterogeneous disease and can be caused by mutations in at least 22 genes (http:// www.sph.uth.tmc.edu/RetNet, accessed as of September 3, 2017). We performed WES on 115 sporadic LCA patients. As LCA is a rare Mendelian disorder, variants with maximum population allele frequency $>0.5 \%$ were filtered out based on the allele frequency in the large public databases of normal populations (i.e., 1000 genome, dbSNP, ESP6500, ExAC, gnomAD) and an internal database. We only focused on rare protein-changing variants including nonsense variants, splicing donor/acceptor variants, missense variants, and small INDELs, since they are more likely to be the disease-causing mutations. One previously simulated control cohort $(n=$ 5000) was used as the control cohort for these tests.

GRIPT showed high sensitivity for the LCA cohort with high locus and ethnicity heterogeneity. It successfully detected the disease gene that only accounted for $\leq$ $1 \%$ of the patients. Specifically, the first nine candidate genes ranked by GRIPT were all known retinal disease genes (Table 1). Among a total of 203 significant candidates, 19 genes were known disease genes, each of which accounted for $0.87-6.09 \%$ (one to seven patients) of the cohort. Most interestingly, GRIPT was able to identify novel retinal disease genes, i.e., POMGNT1 $(p=2.81 \times$ $\left.10^{-10}\right)$ and MFSD8 $\left(p=2.81 \times 10^{-10}\right)$. POMGNT1 was a gene causing non-syndromic RP newly discovered in 2016 [32] and accounted for one patient of this cohort, who carried a stop-gain mutation and a missense mutation in POMGNT1. Mutations in MFSD8 have been linked to macular dystrophy recently [33] and accounted for one patient of the LCA cohort, who carried a splice donor mutation and a missense mutation in MFSD8.

In comparison, the other tools lacked power in detecting the disease genes accounting for small proportions of this cohort. A total of 7 significant candidates were identified by VAAST2, 27 by CMC, 6 by SKAT, and 1 by KBAC. Among them, 5 genes by VAAST2 were known disease genes, 3 genes by CMC, 2 genes by SKAT, and 1 gene by KBAC, each of which accounted for 2.61-6.09\% (three to seven patients) of the cohort. However, none of these known genes were the recently identified novel retinal disease genes.

\section{The RP cohort}

$\mathrm{RP}$ is an inherited retinal disease with even greater genetic heterogeneity compared to LCA. So far, mutations in more than 65 genes were found to cause the disease (http:// www.sph.uth.tmc.edu/RetNet, accessed by September 3, 2017). WES was performed for 154 sporadic RP patients. After filtering, the WES data of the real patient cohort and a simulated control cohort $(n=5000)$ were subjected to analysis. GRIPT again showed excellent power in identifying 
Table 1 Known disease genes were given high ranks and significant $p$ values by GRIPT in a LCA cohort

\begin{tabular}{|c|c|c|c|c|c|c|c|c|c|c|c|}
\hline \multirow[t]{2}{*}{ Genes } & \multirow{2}{*}{$\begin{array}{l}\text { No. of } \\
\text { patients } \\
\text { (\%) }\end{array}$} & \multicolumn{2}{|l|}{ GRIPT } & \multicolumn{2}{|c|}{ VAAST2 } & \multicolumn{2}{|l|}{ CMC } & \multicolumn{2}{|l|}{ SKAT } & \multicolumn{2}{|l|}{ KBAC } \\
\hline & & Rank & $p$ value & Rank & $p$ value & Rank & $p$ value & Rank & $p$ value & Rank & $p$ value \\
\hline NMNAT1 & $4(3.48 \%)$ & 1 & 6.97E-39 & 7 & $2.50 \mathrm{E}-06$ & 12 & $2.52 \mathrm{E}-09$ & 18 & $1.78 \mathrm{E}-04$ & 3 & $1.50 \mathrm{E}-05$ \\
\hline GUCY2D & $6(5.21 \%)$ & 2 & $3.40 \mathrm{E}-32$ & 2 & $2.50 \mathrm{E}-06$ & 102 & 1.15E-03 & 14 & $1.13 \mathrm{E}-04$ & 35 & $1.84 \mathrm{E}-03$ \\
\hline AIPL1 & $3(2.61 \%)$ & 3 & $2.03 E-29$ & 8 & $5.00 \mathrm{E}-06$ & 100 & $1.08 \mathrm{E}-03$ & 24 & $3.01 \mathrm{E}-04$ & 40 & $2.15 E-03$ \\
\hline RPE65 & $3(2.61 \%)$ & 4 & $2.18 \mathrm{E}-29$ & 4 & $2.50 \mathrm{E}-06$ & 16 & $2.44 \mathrm{E}-08$ & 4 & 0 & 2 & $7.00 \mathrm{E}-05$ \\
\hline CEP290 & 7 (6.09\%) & 5 & 1.55E-26 & 1 & $2.50 \mathrm{E}-06$ & 5 & $3.94 \mathrm{E}-11$ & 2 & 0 & 1 & $2.00 \mathrm{E}-05$ \\
\hline CRB1 & 3 (2.61\%) & 6 & $3.41 \mathrm{E}-22$ & 12 & $2.44 \mathrm{E}-05$ & 427 & 0.0231 & 77 & $2.14 \mathrm{E}-03$ & 230 & $2.22 \mathrm{E}-02$ \\
\hline RPGRIP1 & $4(3.48 \%)$ & 7 & $3.41 \mathrm{E}-22$ & 3 & $2.50 \mathrm{E}-06$ & 464 & 0.025 & 164 & $6.30 \mathrm{E}-03$ & 168 & $1.50 \mathrm{E}-02$ \\
\hline SPATA7 & 3 (2.61\%) & 8 & 4.55E-22 & 20 & $1.57 \mathrm{E}-04$ & 1391 & 0.0838 & 534 & $3.16 \mathrm{E}-02$ & 371 & $3.72 \mathrm{E}-02$ \\
\hline TULP1 & $2(1.74 \%)$ & 9 & $6.53 \mathrm{E}-20$ & 2689 & 0.158 & 15,160 & 0.7198 & 879 & 0.06 & 2203 & 0.2324 \\
\hline ADAM9 & $1(0.87 \%)$ & 12 & 7.33E-20 & 325 & 0.0198 & 790 & 0.0483 & 588 & 0.0357 & 243 & 0.0240 \\
\hline IFT140 & $4(3.48 \%)$ & 18 & $5.51 \mathrm{E}-13$ & 499 & 0.0297 & 11,474 & 0.5064 & 3835 & 0.3333 & 7951 & 0.7111 \\
\hline TRNT1 & $1(0.87 \%)$ & 23 & $2.81 E-10$ & 7801 & 0.594 & 17,607 & 0.8925 & 8191 & 0.6 & 5775 & 0.5283 \\
\hline
\end{tabular}

The listed genes are the correctly identified retinal disease genes among the top 20 candidate genes by GRIPT in the LCA cohort. Parameters: 115 cases, 5000 controls, the AR inheritance model

low-frequency disease genes underlying the cohort with high locus and ethnicity heterogeneity. As shown in Table 2, eight genes whose rankings ranged from first to 11th by GRIPT were known retinal disease genes. Among the 157 significant candidates $(p<2.7 \mathrm{e}-6)$ identified by GRIPT, 17 are known disease genes, each of which explained 0.649$8.44 \%$ ( 1 to 13 patients) of the cohort. Furthermore, GRIPT was able to identify three novel retinal disease genes recently published, i.e., POMGNT1 $\left(p=3.95 \times 10^{-15}\right)$, TRNT1 $\left(p=6.25 \times 10^{-8}\right)$ and HGSNAT $\left(p=2.10 \times 10^{-7}\right)$. Mutations in POMGNT1 [32] accounted for two patients of the cohort, who carried two different homozygous missense mutations. Mutations in HGSNAT, a gene causing nonsyndromic RP [34], explained two patients in this cohort. One patient carried two missense mutations, and the other carried a disruptive inframe deletion and a missense mutation. $\mathrm{Mu}$ tations in TRNT1, a gene causing RP and erythrocytic microcytosis [35], accounted for one patient in the cohort, who carried a frameshift mutation and a missense mutation in TRNT1.

In comparison, the other tools had weak power in detecting the low-frequency disease genes underlying this cohort. A total of 4 significant candidate genes were identified by VAAST2, 25 by CMC, 6 by SKAT, and 2 by KBAC. Among them, 2 genes by VAAST2 were known disease genes, 0 by CMC, 1 by SKAT, and 0 by KBAC, each of which accounted for 5.19$8.44 \%$ ( 8 to 13 patients) of the cohort. And none of

Table 2 Known disease genes were given high ranks and significant $p$ values by GRIPT in a RP cohort

\begin{tabular}{|c|c|c|c|c|c|c|c|c|c|c|c|}
\hline \multirow[t]{2}{*}{ Genes } & \multirow{2}{*}{$\begin{array}{l}\text { No. of } \\
\text { patients } \\
(\%)\end{array}$} & \multicolumn{2}{|l|}{ GRIPT } & \multicolumn{2}{|c|}{ VAAST2 } & \multicolumn{2}{|l|}{ CMC } & \multicolumn{2}{|l|}{$\underline{\text { SKAT }}$} & \multicolumn{2}{|l|}{$\mathrm{KBAC}$} \\
\hline & & Rank & $p$ value & Rank & $p$ value & Rank & $p$ value & Rank & $p$ value & Rank & $p$ value \\
\hline$\overline{T U L P 1}$ & $3(1.95 \%)$ & 1 & $2.87 \mathrm{E}-22$ & 15 & 1.57E-04 & 878 & 0.0429 & 279 & 0.0158 & 186 & 0.0190 \\
\hline EYS & 8 (5.19\%) & 2 & 5.67E-18 & 2 & $2.50 \mathrm{E}-06$ & 2718 & 0.1231 & 255 & 0.0143 & 504 & 0.0556 \\
\hline POMGNT1 & 2 (1.30\%) & 5 & $3.95 \mathrm{E}-15$ & 854 & 0.0396 & 12,243 & 0.5391 & 8407 & 0.6 & 7477 & 0.6315 \\
\hline CNGA1 & 2 (1.30\%) & 6 & $3.95 E-15$ & 73 & $2.85 \mathrm{E}-03$ & 18,620 & 0.9801 & 4650 & 0.375 & 4150 & 0.3769 \\
\hline $\mathrm{RDH} 5$ & $2(1.30 \%)$ & 7 & $3.95 \mathrm{E}-15$ & 2430 & 0.119 & 2009 & 0.0924 & 1089 & 0.0769 & 900 & 0.0946 \\
\hline USH2A & 13 (8.44\%) & 9 & $2.27 \mathrm{E}-14$ & 1 & $2.50 \mathrm{E}-06$ & 44 & $6.31 \mathrm{E}-05$ & 6 & 0 & 6 & 0.0001 \\
\hline CRB1 & 3 (1.95\%) & 10 & $3.65 \mathrm{E}-11$ & 114 & 4.66E-03 & 222 & 0.0063 & 428 & 0.028 & 92 & 0.0082 \\
\hline MERTK & 3 (1.95\%) & 11 & $6.20 \mathrm{E}-11$ & 19 & 0.0003 & 6523 & 0.2699 & 76 & 0.0023 & 1325 & 0.1384 \\
\hline BBS4 & $2(1.30 \%)$ & 13 & $8.51 \mathrm{E}-10$ & 645 & 0.0297 & 13,022 & 0.5874 & 1309 & 0.0968 & 2036 & 0.1984 \\
\hline MAK & 1 (0.649\%) & 17 & $6.25 \mathrm{E}-08$ & 1694 & 0.0792 & 13,033 & 0.5874 & 11,162 & 0.75 & 3899 & 0.3573 \\
\hline
\end{tabular}

The listed genes are the correctly identified retinal disease genes among the top 20 candidate genes by GRIPT in the RP cohort. Parameters: 154 cases, 5000 controls, the AR inheritance model 
these known genes were the novel retinal disease genes recently identified.

\section{The CDG cohort}

The CDG cohort was composed of six patients from two families who all carry the pathogenic mutations of PGM3 gene [36, 37]. The WES data were downloaded from dbGaP (phs000809.v1.p1) [36]. Thus, this cohort serves as a real data example of a genetic homogeneous disease with extremely small case cohort size from an independent external source. After filtering and annotation, the real WES data and a simulated control cohort $(n=5000)$ were analyzed by the five tools. GRIPT showed the highest accuracy and efficiency in analyzing this homogeneous external cohort. GRIPT correctly ranked PGM3 first $(p=0)$, taking less than 30 min with one CPU. VAAST2 also ranked PGM3 first $(p=2.50 \times$ $10^{-6}$ ) but took about $6 \mathrm{~h}$ with five parallel CPUs. CMC ranked PGM3 11th $\left(p=3.79 \times 10^{-64}\right)$ and took about $2.5 \mathrm{~h}$ with one CPU. The $p$ value of PGM3 by SKAT equals to 0 but is the same as the other 162 genes $(p=$ 0 ), taking $9.3 \mathrm{~h}$ with one CPU. The $p$ value of PGM3 by KBAC equals to $2 \times 10^{-6}$ but is the same as the other 62 genes $\left(p=2 \times 10^{-6}\right)$, taking $7.8 \mathrm{~h}$ and one CPU.

\section{Discussion}

In this study, we developed a novel computational method named GRIPT for Mendelian disease gene discovery through analyzing the NGS data of patientcontrol cohorts. The null hypothesis of GRIPT is that a non-disease gene should have similar deleterious mutation load in cases and in controls. GRIPT scores and compares the deleterious mutation load of each gene in the genome between patients and controls using a composite Fisher's test and prioritizes the genes that have significant higher deleterious mutation loads in cases than in controls as the candidate disease genes.

Both simulation and real data tests indicate that GRIPT has great sensitivity and specificity and is highly reliable in discovering Mendelian disease genes. For example, as shown in the benchmark of 400 known disease genes, under the AR model, GRIPT ranked the disease gene first in $97.5 \%$ of the simulations for a patient cohort with a size of 600 and with only $1 \%$ of patients carrying the pathogenic mutations of the same gene. In addition, the disease gene was usually the only significant candidate gene identified by GRIPT (Fig. $4 \mathrm{a}-\mathrm{c}$ ). Under the AD model, GRIPT ranked the disease genes in the top three in $93.5 \%$ of the simulations when $2 \%$ of patients (cohort size $=600$ ) were attributed to the same gene (Fig. $4 \mathrm{~d}-\mathrm{f}$ ). The average number of significant candidates was about two. Furthermore, the results from analysis of real patient data were consistent with the benchmark results. For the LCA cohort (size $n=115$ ),
GRIPT was able to systematically and accurately identify 19 disease genes ( 5 genes by VAAST2, 3 genes by CMC, 2 genes by SKAT, and 1 by KBAC). The candidates ranked from first to ninth were all real disease genes. For the RP cohort (size $n=154$ ), GRIPT was able to accurately identify 17 genes ( 2 genes by VAAST2, 0 genes by CMC, 1 gene by SKAT, and 0 by KBAC) with seven of the top 10 candidates being real disease genes. Each of the disease genes identified by GRIPT only accounted for $0.649-8.44 \%$ ( 1 to 13 patients) of patients in the LCA or RP cohort. Moreover, as shown in the simulation, GRIPT reached around 100\% power and always ranked the genes to the top for large patient cohorts (e.g., size $n \geq 300$ ) and/or more homogeneous patients (e.g., the same gene explaining $\geq 3 \%$ of the patients), which was also demonstrated by the analysis of the CGD cohort with a size of six and all attributed to the gene PGM3. Most interestingly, GRIPT was able to discover four newly reported disease genes in the analysis of real patient data. Each of these newly discovered genes only accounted for one or two (0.649-1.3\%) patients in the patient cohort. Overall, GRIPT shows the great power in discovering known and novel Mendelian disease genes. It is especially well suited to analyze diseases with high locus (and ethnicity) heterogeneity, which is a major challenge for solving the underlying genetics mechanisms of Mendelian disorders.

GRIPT is also more robust and significantly less affected by potential confounding factors than other disease gene finders. For example, GRIPT remained powerful for small patient cohorts with high locus heterogeneity. In simulation, under the AR model, for a patient cohort with a size of 100 and only two (2\%) patients carrying the pathogenic mutations of the same gene, the disease gene was ranked on average third by GRIPT with $100 \%$ power. In contrast, the mean ranking of the disease gene by other tools was between $\sim 150$ and $\sim 3300$ and all with $0 \%$ power. This result was also consistent with results from real data as previously discussed. Furthermore, using higher allele frequencies as the variant filtering cutoff, which presumably adds more noise to the analysis, had little impact on the performance of GRIPT under the AR model. In the simulation, for a patient cohort with a size of 600 and with six (1\%) patients attributed to the same gene, as the cutoff of variant frequency filtering increased from 0.5 to $2 \%$, the disease gene was ranked first in $98.89 \%$ of simulations by GRIPT with $100 \%$ power. In comparison, the mean rank of the gene was between 11 and 38 by VAAST2, between 2953 and 4420 by CMC, between 269 and 2095 by SKAT, and between 1306 and 1655 by KBAC, all of which had power below $10 \%$. More importantly, GRIPT is significantly less affected by the combined effect of population stratification and high locus heterogeneity, 
which occur frequently in real data and severely impair the performance of other tools as shown in the simulation and real data analysis. In the simulation of the worst-case scenario where the ethnicity of the patient cohort was completely unmatched by that of the control cohort and with only $1 \%$ of the patient cohort (with a cohort size of 500) attributed to the same disease gene under the AR model, GRIPT ranked the disease gene, on average, 32nd with $100 \%$ power although it generated around 107 significant candidates. In contrast, the mean ranking of the disease gene by other tools was greater than 3500 (power $\leq 20 \%$ ), each of which generated more than 1500 significant candidates. Consistently, the other tools displayed lack of power in the real LCA and RP cohorts with mixed ethnicity and high locus heterogeneity.

The performance advantage of GRIPT might be partly due to that it scores the mutation load of a gene according to the Mendelian inheritance rule. Under the AR model, for each individual, GRIPT only considers/scores genes with at least two variants, which could exclude the false positive signals from the genes merely carrying one pathogenic allele in an individual. Furthermore, Fisher's test built upon the combination of a binomial test and a Wilcoxon rank sum test equipped GRIPT the excellent statistical power for comparing highly skewed distributions of gene score (Fig. 1 and the "Methods" section). In comparison, VAAST/VAAST2, CMC, SKAT, and KBAC take into account the genes carrying at least one variant in an individual. In addition, CMC, SKAT, and KBAC group all the variants within a gene to compute the deleterious mutation load of the gene, which makes genes with a large number of rare variants in case cohort (e.g., benign or due to chance) ranked high and creates false positives. As shown in simulation, this impact on the other tools was more pronounced when the true signal was diluted by high locus heterogeneity and/or was compromised by large background noises, e.g., population stratification (or sequencing platform/variant calling difference) or relaxed cutoff of variant filtering frequency.

Simulation results also suggest that to optimize the performance of GRIPT, the following conditions should be considered. First, as one of the key factors affecting sensitivity is the proportion of patients attributed to the same gene, it is highly desirable to increase the homogeneity of patient cohort. One possible approach is to perform detailed phenotyping and gather the patients who share similar phenotypes and are likely due to mutations in one or a small number of genes. Second, while maintaining the homogeneity of the patient cohort, increasing the patient cohort size can also improve sensitivity. For example, by increasing the patient cohort size from 50 to 100 while maintaining $2 \%$ of patients carrying disease mutations of the same gene under the AR model, the average rank of the disease gene increased from 31 to 3 by GRIPT. Third, using the correct inheritance model when running GRIPT can leverage its power. If the inheritance model of the diseases is unclear, GRIPT should be run using different models, including $\mathrm{AD}, \mathrm{AR}, \mathrm{XD}$, and $\mathrm{XR}$, respectively. Fourth, reduction of the noises in the input variants will improve the outcome. For example, large databases of "normal" populations, e.g., gnomAD and ExAC, should be used to pre-filter variants and remove common benign variants that are unlikely to cause diseases, while filtering with internal databases can reduce the error/bias from the sequencing platforms and variant callers. Furthermore, under different inheritance models, the mutations should be pre-filtered with different frequency cutoffs (for example, the variant filtering frequency for $\mathrm{AD}$ model should be more stringent, namely lower than for AR model). Additionally, removing the genes that are highly mutable but known not causing diseases can reduce noise as well. Fifth, the accuracy of variant function/pathogenicity prediction will also impact the performance of GRIPT. Currently GRIPT applies the well-established integrative allele prediction score, i.e., CADD score, to predict the pathogenicity of variants. However, as the scoring system of GRIPT is flexible, users can easily substitute the CADD score with any other score generated by better algorithms for variant pathogenicity prediction. In the aforementioned analysis, we also used DANN and REVEL scores as the variant score, which generates the consistent results, suggesting the reliability and robustness of the statistic test framework of GRIPT. The rule of thumb for using variant score systems is as follows: (1) the scoring systems should reliably and quantitatively predict the deleteriousness of variants, (2) the scores should be scaled/normalized into a genome-wide ranked score to allow the comparison implemented in the statistic test of GRIPT, and (3) the score system should be comprehensive and cover all the possible SNP and INDEL in the genome.

Although GRIPT does not directly identify pathogenic mutations, by identifying candidate (novel) disease genes, it will dramatically reduce the number of variants to be considered for each patient and therefore greatly facilitate the identification of potential mutations. Once the candidate genes are identified, the causal variants of the genes can be further prioritized with the conventional steps: (1) The individuals carrying at least two (recessive mode) or one (dominant mode) rare variants of the candidate gene should be identified from the patient cohort. (2) Multiple variant effect prediction systems can be applied to estimate and compare deleteriousness of the variants in affecting protein function, mRNA splicing, or other regulation processes of the gene (e.g., CADD score, SIFT, Polyphen, MetaLR/SVM, PROVEAN, REVEL, 
phyloP100way_vertebrate, phastCons100way_vertebrate, ada_score, NNsplice). (3) The Sanger validation and segregation tests of the patients and additional relatives should be performed for the candidate variants.

\section{Conclusions}

In summary, we developed a highly accurate and robust case-control analysis method, GRIPT, for the discovery of Mendelian disease genes. It is especially powerful in detecting disease genes underlying diseases with high locus heterogeneity and is less affected by population stratification. It is also efficient, portable, and flexible. In addition, we generated a WES data simulator which is capable of unbiasedly simulating the WES data of control cohorts with any sample size, sex ratio, and population ethnicity for the usage of GRIPT or other tools. As NGS technology advances (e.g., the decrease in cost and time) and greater amounts of large cohort data become available, we envision that GRIPT will make a significant contribution to the discovery of novel Mendelian disease genes and pave the way for better understanding, diagnosis, prevention, and treatment of Mendelian diseases.

\section{Methods}

\section{Each variant is scored to quantify the deleteriousness}

The hypothesis whether GRIPT test is the deleterious mutation loads of a disease-causing gene is significantly higher in the case cohort than in the control cohort. To quantify the deleteriousness of variants, in this study, we applied Combined Annotation Dependent Depletion (CADD v1.3) score to each variant of each gene in every individual [19]. CADD score is an integrative score derived from the integration of diverse annotations and is highly predictive of molecular functionality and pathogenicity [19]. Higher CADD score indicates more deleteriousness of the mutation. In addition, CADD not only provides integrative prediction scores for SNVs but also for INDELs which are missing for most other variant effect prediction tools. We further normalized the variant score on a scale of 0 to 1 as $s=1-10^{-C / 10}$. $C$ is the PHRED-like scaled $C$-score as described in CADD. Moreover, CADD score can be easily replaced by any other score that users provide in order to better predict the variant's deleteriousness. To test the reliability and robustness of the statistic test framework of GRIPT, the ranked REVEL [30] and DANN [29] scores were also applied as the variant scores respectively. The CADD score (v1.3) was downloaded from https://cadd.gs.washington.edu/ download. The ranked DANN score was extracted from dbNSFP3.4a downloaded from https://sites.google. com/site/jpopgen/dbNSFP. The ranked REVEL score was downloaded from https://sites.google.com/site/ revelgenomics/downloads [38, 39].
Each gene is scored under different inheritance models Under the autosomal recessive (AR) model, only the genes with at least two variants in an individual will be assigned a positive score. The sum of the two highest scores of variants within a gene is used as the score of that gene in the individual. If two variants of a gene are in cis (namely, the two variants reside on the same chromosome) in an individual, only the variant with the higher score will be considered. If a gene carries greater than or equal to one variant in an individual, the score of this gene will be 0 for that individual. Under the AR model, the maximum score for a gene is 2 , and the minimum is 0 .

Under the autosomal dominant (AD) model, only the genes with at least one variant in an individual will be assigned a positive score. The highest score of variants within a gene is used as the score of that gene in the individual. Under the AD model, the maximum score for a gene is 1 , and the minimum is 0 .

Similarly, under the $\mathrm{X}$-linked recessive model, the sum of the two highest variant scores is used as the score of each gene on the $\mathrm{X}$ chromosome in an individual. And under the X-linked dominant model, the highest variant score is used as the score of each gene on the $\mathrm{X}$ chromosome in an individual.

\section{Gene score distribution is highly skewed for rare Mendelian disorders}

As mentioned above, each gene has a score in each case or control individual, ranging from 0 to 1 (for dominant models) or 2 (for recessive models). Then, for each gene, we compare the gene score distribution in the case cohort to that in the control cohort. The null hypothesis is that the deleterious mutation load of a gene is not significantly different between cases and controls. Thus, the significance of the one-tailed alternative hypothesis that the deleterious mutation load is higher in cases than controls could suggest the likelihood of the gene associated with the disease.

To choose the appropriate statistic test, we first characterized the gene score distribution. We found that the score distributions of most genes are highly skewed with excesses of zeros. This is expected mainly because Mendelian diseases are rare and so are the disease-causing mutations. Usually, after filtering out known common human variants which are likely benign, only a small number of rare variants (e.g., $\mathrm{MAF} \leq 0.5 \%$ ) in cases and controls will be kept. Moreover, among the filtered rare variants, only some of them have deleterious effects; therefore, only these rare, deleterious variants will have positive variant scores. In addition, the recessive model requires a biallelic state to assign a positive gene score in one individual. Thus, the scores of a gene in most case individuals and control individuals are zeros. An example of 
USH $2 \mathrm{~A}$ gene score distributions in our retinal disease patient cohort $(n=250)$ and an internal control cohort $(n=$ $250)$ is shown in Fig. 2.

Combining two separate statistical tests with Fisher's test To compare the highly skewed distributions of gene scores in the case and control cohorts derived above, we test a composite null hypothesis by applying Fisher's test to combine two separate tests including a binomial test and a Wilcoxon rank sum test [20]. The composite null hypothesis is designed to answer two questions. The first question is whether the proportions of non-zero scores are similar in the case cohort and control cohort $\left(Z_{1}=\right.$ $0)$. The second question is whether the values of non-zero scores are similar in the case cohort and control cohort $\left(Z_{2}=0\right)$. Namely, Fisher's method will test the $\mathrm{H}_{0}: Z_{1}=0$ and $Z_{2}=0$ versus the one-tailed alternative $\mathrm{H}_{1}: Z_{1}>0$ and/or $Z_{2}>0$ [20].

Let $N_{1}$ and $N_{2}$ be the total number of cases and controls, respectively. Let $n_{1}$ and $n_{2}$ be the number of non-zero score in cases and controls, respectively.

The first statistic, $Z_{1}$, represents the proportion difference of non-zero scores between cases and controls. Given $n_{1}+n_{2}=n$ and $r=N_{2} / N_{1}, n_{1}$ is approximately distributed as $\operatorname{Binomial}\left(n,(1+r)^{-1}\right)$ under $\mathrm{H}_{0}$. Hence, a one-tailed $p$ value $p_{1}$ can be obtained as the tail area under the $N(0,1)$ p.d.f to the right of

$$
Z_{1}=\frac{\frac{n_{1}}{n_{1}+n_{2}}-\frac{1}{1+r}}{\sqrt{\frac{r}{(1+r)(1+r)\left(n_{1}+n_{2}\right)}}}
$$

The second statistic, $Z_{2}$, represents the difference of the non-zero scores between cases and controls. The standardized Wilcoxon rank sum test was applied to test whether the gene cores in cases are significantly higher than those in controls. Let $p_{2}$ denote the corresponding one-tailed $p$ value.

Finally, Fisher's method is used to test the composite null hypothesis $\mathrm{H}_{0}: Z_{1}=0$ and $Z_{2}=0$ at one-tailed level $\alpha$ based on a combination of $Z_{1}$ and $Z_{2}$ or $p_{1}$ and $p_{2}$ as follows:

Reject $\mathrm{H}_{0}$ if $p<\alpha$, where $p=P\left(\chi_{4}^{2}>-4 \log _{e} \sqrt{p_{1} p_{2}}\right)$.

Here, $\chi_{4}^{2}$ is a $\chi^{2}$ distribution with 4 d.f.; therefore, the $p$ value can be calculated using a $\chi^{2}$ distribution.

The program of GRIPT is written in Java and $R$.

\section{A WES data simulator based on ExAC database}

The VCF file of ExAC database (ExAC.r0.3.1.sites.vep.vcf) was downloaded from http://exac.broadinstitute.org/downloads [21]. We collected the variants recorded in the VCF file which were not indicated as filtered by ExAC. For each of these variants, we extracted information on the genomic position, the allele count, the chromosome number, and the allele frequency in each subpopulation, including AFR (African/African American), AMR (American), EAS (East Asian), FIN (Finnish), NFE (Non-Finnish European), SAS (South Asian), OTH (Other), adjusted population, and raw data. We only considered the ExAC variants that were missense or loss-of-function mutations (e.g., missense mutations, stop-gained mutations, splicing donor/acceptor mutations, and frameshift mutations). We also downloaded the CADD scores v1.3 for the ExAC variants from http://cadd.gs.washington.edu/download [19] and annotated each collected ExAC variant with its corresponding CADD score. Next, we wrote a WES data simulator program in PERL. Briefly, the script simulated the WES data per person individually. For each individual, the simulator will go through the variants recorded in the ExAC database which satisfy the variant filtering criteria (e.g., MAF $\leq 0.5 \%$ ) one by one and output the reference nucleotide or the altered nucleotide according to the allele frequency of that variant in ExAC. For example, in the position chr1:10000, if the allele frequency of " $\mathrm{A}>\mathrm{T}$ " is $0.2 \%$ and the allele frequency of " $\mathrm{A}>\mathrm{G}$ " is $0.5 \%$, then in the simulated WES data of one person, there is $0.2 \%$ of chances the simulator will output the SNP "A > T," $0.5 \%$ of chances will output the SNP "A > G," and $99.3 \%$ of chances the simulator will generate "A > A," namely no SNP ouput in chr1:10000. Thus, each generated variant follows a multinomial distribution according to its frequency in the user-selected ethnic population based on the ExAC database. For a given number $(N)$ of individuals with a given sex ratio, the simulator will generate "N" WES data files individually. Each WES data file includes information such as reference nucleotides, altered nucleotides, the coordinates in the genome, and the CADD scores of the variants.

\section{Simulation of patient and control cohorts}

To evaluate the performance of GRIPT, we performed the simulation tests on GRIPT and similar tools, i.e., VAAST2, CMC, SKAT, and KBAC. The WES data of the patient cohort and control cohort were first generated using the WES data simulator mentioned above. Given the rare frequency of Mendelian disease-causing variants in normal population, for the AR model, the WES data were simulated based on the variants whose maximum population frequency was $\leq 0.5 \%$ in ExAC database by default, while for the AD model, based on the variants whose maximum population frequency was $\leq 0.01 \%$ in ExAC database by default, unless otherwise specified. We used "adjusted" average population frequency as the default variant frequency, unless otherwise specified. Then, we randomly selected pathogenic variants of a given disease gene from HGMD database with MAF $\leq$ 
0.5\% in ExAC database and inserted them into a given percentage of individuals randomly selected from the patient cohort to mimic the patient cohort with genetic heterogeneity. In the AR model, two variants were respectively selected from HGMD and spiked into each selected individual. Thus, the two variants spiked into the same individual can be the same (homozygous) or different (heterozygous). In the AD model, only one variant was randomly selected and spiked into each selected individual. Therefore, under the AR or AD model, the pathogenic mutations of a given gene can be the same or different within and between the patients. No additional mutations were spiked into the control cohort. For each spike in percentage level per scenario, 30 simulation runs were repeated (Additional file 2: Figure S5).

\section{The implementation of VAAST2, CMC, SKAT, KBAC, and Fisher's exact test}

The latest release of VAAST2 was obtained from http:// www.yandell-lab.org/software/vaast.html [16, 17]. The CMC, SKAT, and KBAC were implemented through the "Rvtests" software package downloaded from https:// genome.sph.umich.edu/wiki/Rvtests\#Download [22]. The $p$ values of VAAST2, SKAT, and KBAC were obtained using 400,000 permutations. Fisher's exact test was implemented through the PLINK v1.90b5.2 package from https://www.cog-genomics.org/plink/1.9/ [40]. The intermediate steps were carried out using PERL and $R$ scripts.

\section{Preprocessing the variants in cis}

To reduce false positive, we recommend the users to handle the variants in cis before inputting data into GRIPT. However, given that it is not always possible to obtain accurate phasing information, GRIPT can tolerate imperfect phasing as shown in the aforementioned simulation and real data analyses. Currently, a preprocessing script included in the GRIPT package was used to handle variants in cis, which performs the following operations:

1) If the genomic coordinates of two variants are within 100 bp, Fisher's exact test will be performed to determine whether the two variants are in cis by comparing the ratio of the variant base sequencing coverage to the reference base sequencing coverage of the two variants. If the two variants are in cis and within $100 \mathrm{bp}$, they can be covered by a large number of the same sequencing reads; therefore, their read coverage ratios would be similar and Fisher's exact test $p$ value would be large. In contrast, if they are in trans and close to each other, they would be covered by different sequencing reads; thus, the read coverage ratios of the two variants would be different and Fisher's exact test $p$ value would be small. We take Fisher's exact test $p \geq 0.4$ as the cutoff to deduce the read coverage ratios of the two variants are similar, namely, the two variants as in cis, otherwise as in trans. Using a different $p$ value cutoff does not significantly impact on the result. For example, we have used $p<0.05$ as the cutoff to assign the variants in trans and $p \geq 0.05$ to assign the two variants in cis. Although this could mistakenly assign a few in trans variants as in cis, the results remained consistent. Because GRIPT is built on the mutation burden in the case cohort and control cohort but not a single case, a few imperfect phasing cases can be tolerated. If the two variants are determined to be in cis by Fisher's test, the variant with higher variant score (e.g., CADD score) will be passed on to the subsequent analysis, while the one with lower variant score will be ignored.

2) For each gene in every individual, all variants within the gene will be searched against the same gene in the rest of the individuals of the case cohort. If a gene has $\geq 2$ variants present concurrently in $\geq 2$ individuals, it is likely that these variants are in cis. Because given the sample size of case cohorts $(n=$ 115 for LCA, 154 for the RP cohort, and currently available case cohort size mostly $\leq 5000$ ) and the rare frequency of Mendelian disease-causing mutations (allele frequency $\leq 0.5 \%$ ), the chance for two or more rare variants co-occurring in unrelated individuals is very small $\left(5000 \times(0.005 \times 0.005)^{\wedge}{ }^{2} \ll\right.$ $2)$, unless these variants are in cis or the disease is specifically caused by the combination of the variants. Although our preprocessing script does not fit the latter situation, it can help clean up the former one. If a gene has $\geq 2$ variants co-occurring in $\geq$ 2 individuals, among the concurrent variants, the script will only keep the variant with the highest variant score and ignore the other concurrent variants in the subsequent analysis.

\section{Additional files}

Additional file 1: Table S1. The sensitivity and specificity of GRIPT with CADD and other tests under the AR and AD models. Table S2. Benchmark on 400 randomly selected known disease genes. Table S3. Test the effect of the patient cohort sample size. Table $\mathbf{S 4}$. Test the effect of population stratification in cohorts. Table S5. Test the effect of variant frequency filtering. Table S6. Test the effect of the control cohort size. Table S7. The performance of detecting genes with modest-effect variants. (XLSX $111 \mathrm{~kb}$ )

Additional file 2: Figure S1. Benchmark of GRIPT with CADD, REVEL, and DANN scores on 400 Mendelian disease genes. Figure S2. Test the impact of patient cohort sizes with REVEL and DANN scores. Figure S3. Test the impact of population stratification with REVEL and DANN scores. Figure S4. Test the impact of variant frequency filtering with REVEL and DANN scores. Figure S5. The main procedure of simulation analysis. (PDF 3207 kb) 


\section{Abbreviations}

AD: Autosomal dominant; AFR: African/African American; AMR: American; AR: Autosomal recessive; CADD: Combined Annotation Dependent Depletion; CAST: Cohort Allelic Sums Test; CMC: Combined Multivariate and Collapsing; DANN: Deleterious Annotation of genetic variants using Neural Networks; EAS: East Asian; ExAC: Exome Aggregation Consortium; FIN: Finnish; gnomAD: Genome Aggregation Database; GRIPT: Gene Ranking, Identification and Prediction Tool; GWSL: Genome-wide significant level; HGMD: Human Gene Mutation Database; INDEL: Insertion and deletion; KBAC: Kernel-Based Adaptive Clustering; LCA: Leber's congenital amaurosis; NFE: Non-Finnish European; NGS: Next-generation sequencing; NSAG: Number of significant autosomal genes; OMIM: Online Mendelian Inheritance in Man; OTH: Other; REVEL: Rare Exome Variant Ensemble Learner; RP: Retinitis pigmentosa; SAS: South Asian; SKAT: Sequence Kernel Association Test; SNP: Single nucleotide polymorphism; VAAST: Variant Annotation, Analysis and Search Tool; VCF: Variant Call Format; WES: Whole exome sequencing; WGS: Whole genome sequencing; WRST: Wilcoxon rank sum test; XD: X-linked dominant; XR: X-linked recessive

\section{Acknowledgements}

We thank all the patients for participating in our study. Funding for the study of Autosomal Recessive PGM3 Mutations Cause a New Congenital Disorder of Glycosylation was provided in part by Merck Research Laboratories and by the Intramural Research Program of the NIAID. The datasets used for the analyses of CDG cohort described in this manuscript were obtained from dbGaP at https://www.ncbi.nlm.nih.gov/projects/gap/ cgi-bin/study.cgi?study_id=phs000809.v1.p1 through dbGaP study accession numbers phs000809.v1.p1. Data from the Autosomal Recessive PGM3 Mutations Cause a New Congenital Disorder of Glycosylation Project were provided by Dr. Su and Dr. Milner on behalf of his collaborators at NIAID.

\section{Funding}

This work was supported by grants from the National Eye Institute (R01EY022356, R01EY018571, EY002520) and Retinal Research Foundation, and $\mathrm{NIH}$ shared instrument grant S100D023469 to RC. For FW, this work was supported by grants from National Natural Science Foundation of China (61472086 and 81728005) and grants from National Key Research and Development Program of China (2016YFC0902100). For JW, this work was supported by the Career Starter Research Grant of Knights Templar Eye Foundation.

\section{Availability of data and materials}

The GRIPT software, scripts, WES data simulator, and test examples are distributed under the GNU General Public License v3.0 and can be downloaded from Github: https://github.com/fe4960/GRIPT_BCM [41] or zenodo: https://doi.org/10.5281/zenodo.1407225 with the DOl:https:// doi.org/10.5281/zenodo.1407225 [42]. The simulated datasets can be downloaded from zenodo: https://doi.org/10.5281/zenodo.1475305 with the DOl: https://doi.org/10.5281/zenodo.1475305 [43]. The datasets used for the analyses of CDG cohort described in this manuscript were obtained from dbGaP at https://www.ncbi.nlm.nih.gov/projects/gap/cgi-bin/

study.cgi?study_id=phs000809.v1.p1 through dbGaP study accession number phs000809.v1.p1 [36, 37]. The datasets used for the analyses of LCA and RP cohorts can be available from dbGaP at https://www.ncbi.nlm.nih.gov/ projects/gap/cgi-bin/study.cgi?study_id=phs001517.v1.p1 with dbGaP study accession number phs001517.v1.p1 [44].

\section{Authors' contributions}

$J W, L Z, X W, F W$, and RC proposed the method. JW, LZ, XW, and YC wrote the software. JW, LZ, and $Y C$ performed the simulation test. JW, MX, ZG, and ZS analyzed the patient data. JW, LZ, XW, PW, and RC wrote the manuscript. All authors read and approved the final manuscript.

\section{Ethics approval and consent to participate}

All subjects in the LCA and RP study underwent ophthalmic evaluations. Informed consent was obtained from all patients or their guardians. All the diagnostic procedures were approved by the local institutional review boards or ethics committees. Baylor College of Medicine IRB assurance number is 00000286 . All experimental methods comply with the Helsinki Declaration.

\section{Consent for publication}

All patients or their guardians have given written informed consent for publication.

\section{Competing interests}

The authors declare that they have no competing interests.

\section{Publisher's Note}

Springer Nature remains neutral with regard to jurisdictional claims in published maps and institutional affiliations.

\section{Author details}

'Human Genome Sequencing Center, Baylor College of Medicine, Houston, TX 77030, USA. ${ }^{2}$ Department of Molecular and Human Genetics, Baylor College of Medicine, Houston, TX 77030, USA. ${ }^{3}$ Structural and Computational Biology and Molecular Biophysics Graduate Program, Baylor College of Medicine, Houston, TX 77030, USA. " Baylor Miraca Genetics Laboratories, Houston, TX 77030, USA. ${ }^{5}$ Shanghai Key Lab of Intelligent Information Processing, School of Computer Science and Technology, Fudan University, Shanghai, China.

Received: 15 May 2018 Accepted: 2 November 2018

Published online: 26 November 2018

\section{References}

1. Baird PA, Anderson TW, Newcombe HB, Lowry RB. Genetic disorders in children and young adults: a population study. Am J Hum Genet. 1988;42: 677-93.

2. Christianson A, Howson CP, Modell B. March of Dimes Global Report on birth defects: the hidden toll of dying and disabled children. In: March of Dimes Birth Defects Foundation; 2006. https://www.marchofdimes.org/ materials/global-report-on-birth-defects-the-hidden-toll-of-dying-anddisabled-children-executive-summary.pdf.

3. Guttmacher AE, Collins FS. Genomic medicine-a primer. N Engl J Med. 2002;347:1512-20.

4. Zhao L, Wang F, Wang H, Li Y, Alexander S, Wang K, Willoughby CE, Zaneveld JE, Jiang L, Soens ZT, et al. Next-generation sequencing-based molecular diagnosis of 82 retinitis pigmentosa probands from Northern Ireland. Hum Genet. 2015;134:217-30.

5. Wang H, den Hollander Al, Moayedi Y, Abulimiti A, Li Y, Collin RW, Hoyng CB, Lopez I, Abboud EB, Al-Rajhi AA, et al. Mutations in SPATA7 cause Leber congenital amaurosis and juvenile retinitis pigmentosa. Am J Hum Genet. 2009:84:380-7.

6. Vithana EN, Abu-Safieh L, Allen MJ, Carey A, Papaioannou M, Chakarova C, Al-Maghtheh M, Ebenezer ND, Willis C, Moore AT, et al. A human homolog of yeast pre-mRNA splicing gene, PRP31, underlies autosomal dominant retinitis pigmentosa on chromosome 19q13.4 (RP11). Mol Cell. 2001;8:375-81.

7. Bamshad MJ, Shendure JA, Valle D, Hamosh A, Lupski JR, Gibbs RA, Boerwinkle E, Lifton RP, Gerstein M, Gunel M, et al. The Centers for Mendelian Genomics: a new large-scale initiative to identify the genes underlying rare Mendelian conditions. Am J Med Genet A. 2012;158A:1523-5.

8. Alkuraya FS. Homozygosity mapping: one more tool in the clinical geneticist's toolbox. Genet Med. 2010;12:236-9.

9. Ku CS, Naidoo N, Pawitan Y. Revisiting Mendelian disorders through exome sequencing. Hum Genet. 2011;129:351-70.

10. Chong JX, Buckingham KJ, Jhangiani SN, Boehm C, Sobreira N, Smith JD, Harrell TM, McMillin MJ, Wiszniewski W, Gambin T, et al. The genetic basis of Mendelian phenotypes: discoveries, challenges, and opportunities. Am J Hum Genet. 2015;97:199-215.

11. Li B, Leal SM. Methods for detecting associations with rare variants for common diseases: application to analysis of sequence data. Am J Hum Genet. 2008;83:311-21.

12. Morgenthaler S, Thilly WG. A strategy to discover genes that carry multiallelic or mono-allelic risk for common diseases: a cohort allelic sums test (CAST). Mutat Res. 2007;615:28-56.

13. Madsen BE, Browning SR. A groupwise association test for rare mutations using a weighted sum statistic. PLoS Genet. 2009;5:e1000384.

14. Wu MC, Lee S, Cai T, Li Y, Boehnke M, Lin X. Rare-variant association testing for sequencing data with the sequence kernel association test. Am J Hum Genet. 2011;89:82-93. 
15. Liu DJ, Leal SM. A novel adaptive method for the analysis of nextgeneration sequencing data to detect complex trait associations with rare variants due to gene main effects and interactions. PLoS Genet. 2010;6: e1001156.

16. Yandell M, Huff C, Hu H, Singleton M, Moore B, Xing J, Jorde LB, Reese MG A probabilistic disease-gene finder for personal genomes. Genome Res. 2011;21:1529-42

17. Hu H, Huff CD, Moore B, Flygare S, Reese MG, Yandell M. VAAST 2.0: improved variant classification and disease-gene identification using a conservation-controlled amino acid substitution matrix. Genet Epidemiol. 2013:37:622-34.

18. Amberger JS, Bocchini CA, Schiettecatte F, Scott AF, Hamosh A. OMIM.org: Online Mendelian Inheritance in Man $\left(\mathrm{OMIM}^{\oplus}\right)$, an online catalog of human genes and genetic disorders. Nucleic Acids Res. 2015;43:D789-98.

19. Kircher M, Witten DM, Jain P, O'Roak BJ, Cooper GM, Shendure J. A general framework for estimating the relative pathogenicity of human genetic variants. Nat Genet. 2014;46:310-5.

20. Mehrotra DV, Li X, Gilbert PB. A comparison of eight methods for the dualendpoint evaluation of efficacy in a proof-of-concept HIV vaccine trial. Biometrics. 2006;62:893-900.

21. Lek M, Karczewski KJ, Minikel EV, Samocha KE, Banks E, Fennell T, O'DonnellLuria AH, Ware JS, Hill AJ, Cummings BB, et al. Analysis of protein-coding genetic variation in 60,706 humans. Nature. 2016;536:285-91.

22. Zhan X, Hu Y, Li B, Abecasis GR, Liu DJ. RVTESTS: an efficient and comprehensive tool for rare variant association analysis using sequence data. Bioinformatics. 2016;32:1423-6.

23. Marlhens F, Bareil C, Griffoin JM, Zrenner E, Amalric P, Eliaou C, Liu SY, Harris E, Redmond TM, Arnaud B, et al. Mutations in RPE65 cause Leber's congenital amaurosis. Nat Genet. 1997;17:139-41.

24. Gu SM, Thompson DA, Srikumari CR, Lorenz B, Finckh U, Nicoletti A, Murthy KR, Rathmann M, Kumaramanickavel G, Denton MJ, Gal A. Mutations in RPE65 cause autosomal recessive childhood-onset severe retinal dystrophy. Nat Genet. 1997;17:194-7.

25. Morimura H, Fishman GA, Grover SA, Fulton AB, Berson EL, Dryja TP. Mutations in the RPE65 gene in patients with autosomal recessive retinitis pigmentosa or leber congenital amaurosis. Proc Natl Acad Sci U S A. 1998;95:3088-93.

26. Savage SA, Giri N, Baerlocher GM, Orr N, Lansdorp PM, Alter BP. TINF2, a component of the shelterin telomere protection complex, is mutated in dyskeratosis congenita. Am J Hum Genet. 2008;82:501-9.

27. Walne AJ, Vulliamy $T$, Beswick R, Kirwan M, Dokal I. TINF2 mutations result in very short telomeres: analysis of a large cohort of patients with dyskeratosis congenita and related bone marrow failure syndromes. Blood. 2008;112: 3594-600.

28. Sasa GS, Ribes-Zamora A, Nelson ND, Bertuch AA. Three novel truncating TINF2 mutations causing severe dyskeratosis congenita in early childhood. Clin Genet. 2012;81:470-8.

29. Quang D, Chen Y, Xie X. DANN: a deep learning approach for annotating the pathogenicity of genetic variants. Bioinformatics. 2015;31:761-3.

30. Ioannidis NM, Rothstein JH, Pejaver V, Middha S, McDonnell SK, Baheti S, Musolf A, Li Q, Holzinger E, Karyadi D, et al. REVEL: an ensemble method for predicting the pathogenicity of rare missense variants. Am J Hum Genet. 2016;99:877-85.

31. Zernant J, Lee W, Collison FT, Fishman GA, Sergeev YV, Schuerch K, Sparrow JR, Tsang SH, Allikmets R. Frequent hypomorphic alleles account for a significant fraction of ABCA4 disease and distinguish it from age-related macular degeneration. J Med Genet. 2017;54:404-12.

32. Xu M, Yamada T, Sun Z, Eblimit A, Lopez I, Wang F, Manya H, Xu S, Zhao L, $\mathrm{Li} Y$, et al. Mutations in POMGNT1 cause non-syndromic retinitis pigmentosa. Hum Mol Genet. 2016;25:1479-88.

33. Roosing S, van den Born LI, Sangermano R, Banfi S, Koenekoop RK, Zonneveld-Vrieling MN, Klaver CC, van Lith-Verhoeven JJ, Cremers FP, den Hollander Al, Hoyng CB. Mutations in MFSD8, encoding a lysosomal membrane protein, are associated with nonsyndromic autosomal recessive macular dystrophy. Ophthalmology. 2015;122:170-9.

34. Haer-Wigman L, Newman H, Leibu R, Bax NM, Baris HN, Rizel L, Banin E, Massarweh A, Roosing S, Lefeber DJ, et al. Non-syndromic retinitis pigmentosa due to mutations in the mucopolysaccharidosis type IIIC gene, heparan-alpha-glucosaminide N-acetyltransferase (HGSNAT). Hum Mol Genet. 2015;24:3742-51.

35. DeLuca AP, Whitmore SS, Barnes J, Sharma TP, Westfall TA, Scott CA, Weed MC, Wiley JS, Wiley LA, Johnston RM, et al. Hypomorphic mutations in
TRNT1 cause retinitis pigmentosa with erythrocytic microcytosis. Hum Mol Genet. 2016:25:44-56.

36. Zhang Y, Yu X, Ichikawa M, Lyons JJ, Datta S, Lamborn IT, Jing H, Kim ES, Biancalana M, Wolfe LA, et al. Autosomal recessive phosphoglucomutase 3 (PGM3) mutations link glycosylation defects to atopy, immune deficiency, autoimmunity, and neurocognitive impairment. J Allergy Clin Immunol. 2014:133:1400-9 1409 e1401-1405.

37. Hay BN, Martin JE, Karp B, Davis J, Darnell D, Solomon B, Turner M, Holland SM, Puck JM. Familial immunodeficiency with cutaneous vasculitis, myoclonus, and cognitive impairment. Am J Med Genet A. 2004;125A:145-51.

38. Liu X, Jian X, Boerwinkle E. dbNSFP: a lightweight database of human nonsynonymous SNPs and their functional predictions. Hum Mutat. 2011;32: 894-9.

39. Liu X, Wu C, Li C, Boerwinkle E. dbNSFP v3.0: a one-stop database of functional predictions and annotations for human nonsynonymous and splice-site SNVs. Hum Mutat. 2016;37:235-41.

40. Chang CC, Chow CC, Tellier LC, Vattikuti S, Purcell SM, Lee JJ. Secondgeneration PLINK: rising to the challenge of larger and richer datasets. Gigascience. 2015;4:7.

41. Wang J, Zhao L, Wang X, Chen Y, Xu M, Soens Z, Ge Z, Wang P, Wang F, Chen R. GRIPT: a novel case-control analysis method for Mendelian disease gene discovery. Github. 2018; https://github.com/fe4960/GRIPT_BCM. Accessed 16 Nov 2018.

42. Wang J, Zhao L, Wang X, Chen Y, Xu M, Soens Z, Ge Z, Wang P, Wang F, Chen R. The source code and scripts of GRIPT package: a novel case-control analysis method for Mendelian disease gene discovery. Zenodo. 2018. https://doi.org/10.5281/zenodo.1407225. Accessed 16 Nov 2018.

43. Wang J, Zhao L, Wang X, Chen Y, Xu M, Soens Z, Ge Z, Wang P, Wang F, Chen R. The simulation dataset of GRIPT: a novel case-control analysis method for Mendelian disease gene discovery. Zenodo. 2018; https://doi. org/10.5281/zenodo.1475305. Accessed 16 Nov 2018.

44. Chen R. Genetics of Human Inherited Retinal Diseases (GHIRD). dbGaP. https://www.ncbi.nlm.nih.gov/projects/gap/cgi-bin/study.cgi?study_id= phs001517.v1.p1. Accessed 16 Nov 2018.

Ready to submit your research? Choose BMC and benefit from

- fast, convenient online submission

- thorough peer review by experienced researchers in your field

- rapid publication on acceptance

- support for research data, including large and complex data types

- gold Open Access which fosters wider collaboration and increased citations

- maximum visibility for your research: over $100 \mathrm{M}$ website views per year

At BMC, research is always in progress.

Learn more biomedcentral.com/submissions 